\title{
Natural Bioactive Molecules as Potential Agents Against SARS-CoV-2
}

\author{
Wei Chen ${ }^{1}$, Zhihao Wang ${ }^{2}$, Yawen Wang ${ }^{2,3}$ and Yiping $L i^{1 *}$ \\ ${ }^{1}$ Department of Medicinal Chemistry, School of Pharmacy, Xi'an Jiaotong University, Xi'an, China, ${ }^{2}$ Biobank, First Affiliated \\ Hospital of Xi'an Jiaotong University, Xi'an, China, ${ }^{3}$ Department of Laboratory Medicine, First Affiliated Hospital of Xi'an Jiaotong \\ University, Xi'an, China
}

OPEN ACCESS

Edited by: Ilaria Peluso,

Council for Agricultural and Economics Research (CREA), Italy

Reviewed by: Lalith Perera, National Institute of Environmental Health Sciences (NIEHS), United States Simone Carradori, University "G. d'Annunzio" of ChietiPescara, Italy

*Correspondence: Yiping $\mathrm{Li}$ yipingli@mail.xjtu.edu.cn

Specialty section: This article was submitted to Ethnopharmacology,

a section of the journal Frontiers in Pharmacology

Received: 29 April 2021 Accepted: 12 July 2021 Published: 17 August 2021

Citation:

Chen W, Wang Z, Wang Y and Li Y (2021) Natural Bioactive Molecules as Potential Agents

Against SARS-CoV-2.

Front. Pharmacol. 12:702472.

doi: 10.3389/fphar.2021.702472
In the past two decades, pandemics of several fatal coronaviruses have posed enormous challenges for public health, including SARS-CoV (2003), MERS-CoV (2012), and SARSCoV-2 (2019). Among these, SARS-CoV-2 continues to ravage the world today and has lead to millions of deaths and incalculable economic damage. Till now, there is no clinically proven antiviral drug available for SARS-CoV-2. However, the bioactive molecules of natural origin, especially medicinal plants, have been proven to be potential resources in the treatment of SARS-CoV-2, acting at different stages of the viral life cycle and targeting different viral or host proteins, such as $P L^{\text {pro }}, 3 \mathrm{CL}^{\text {pro }}, \mathrm{RdRp}$, helicase, spike, $\mathrm{ACE} 2$, and TMPRSS2. They provide a viable strategy to develop therapeutic agents. This review presents fundamental biological information on SARS-CoV-2, including the viral biological characteristics and invasion mechanisms. It also summarizes the reported natural bioactive molecules with anti-coronavirus properties, arranged by their different targets in the life cycle of viral infection of human cells, and discusses the prospects of these bioactive molecules for the treatment of COVID-19.

Keywords: coronavirus, COVID-19, SARS-CoV-2, antiviral agents, natural bioactive molecules, therapeutic targets

\section{INTRODUCTION}

Coronaviruses, which cause respiratory tract infections in mammals and birds, are a group of enveloped, single-stranded, positive-sense RNA viruses that consist of the second-largest RNA genome (26-32 kb) only after planarian secretory cell nidovirus (PSCNV) (41.1 kb) to date (Ziebuhr, 2004; Saberi et al., 2018). Based on different antigenic cross-reactivity and genetic composition, the 26 known coronavirus species are classified into four genera (Alphacoronavirus, Betacoronavirus, Deltacoronavirus, and Gammacoronavirus) $-\alpha$ and $\beta$ genera contain strains that are pathogenic to humans (Cleri et al., 2010). Before December 2019, six of the known coronaviruses, namely, HCoV229E ( $\alpha-\mathrm{CoV}), \mathrm{HCoV}-\mathrm{NL} 63(\alpha-\mathrm{CoV}), \mathrm{HCoV}-\mathrm{OC} 43(\beta-\mathrm{CoV}), \mathrm{HCoV}-\mathrm{HKU} 1(\beta-\mathrm{CoV})$, SARS-CoV $(\beta-\mathrm{CoV})$, and MERS-CoV $(\beta-\mathrm{CoV})$, were reported to cause diseases in humans (Arabi et al., 2017; Skariyachan et al., 2019). The first four have caused localized epidemics where patients exhibited primarily mild and self-limiting symptoms, whereas the last two can cause diseases with severe symptoms and have swept parts of the world in 2003 and 2012, respectively (Hui, 2017; Paules et al., 2020). In January 2020, another novel coronavirus, severe acute respiratory syndrome coronavirus 2 (SARS-CoV-2), was identified as the seventh coronavirus contagious to humans and had been characterized as a new member of the $\beta$-coronavirus genus (Figure 1A) (Lu et al., 2020; Wu et al., 2020).

Coronavirus disease 2019 (COVID-19) has been sweeping the world since its initial detection in Wuhan, China, in December 2019. The COVID-19 pandemic has lead to unprecedented uncertainty 

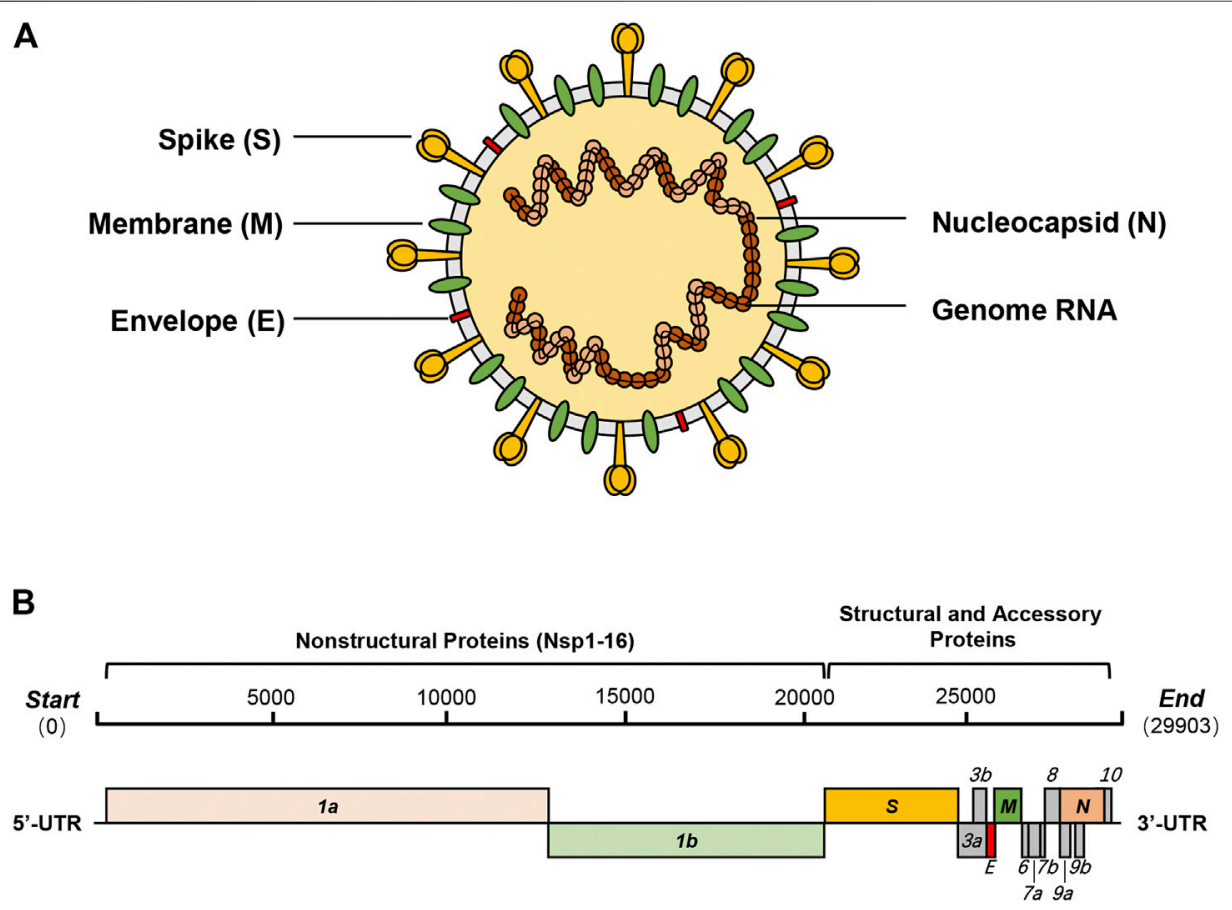

FIGURE 1 | SARS-CoV-2 and its genome organization. (A) SARS-CoV-2 is a new $\beta$-coronavirus with a single-strand, positive-sense RNA genome, which binds with nucleocapsid proteins $(\mathrm{N})$ to form the nucleocapsid. The trimeric spike glycoprotein $(\mathrm{S})$ is a crucial component of virus recognition to host cells. Membrane protein (M) and envelope protein (E) play key roles in the assembly and release of virions. (B) SARS-CoV-2 contains a 29.9 kb genome encoding 14 ORFs. About two-thirds of the genome encodes two polyproteins (pp1a and pp1ab), which are auto-proteolytically processed into 16 non-structural proteins (Nsp1-16). The remaining onethird of the genome encodes four essential structural proteins and nine accessory proteins.

to modern human civilization and unforeseeable changes to global society. With more than 100 million confirmed cases and more than four million deaths, the world encounters economic contraction and the global economy declines. Many countries resorted to stringent social distancing measures to contain the spread of the virus, which included canceling group activities and limiting the movement of people. Meanwhile, numerous efforts have been devoted to SARSCoV-2 studies, including biological characteristics, infection mechanisms, vaccine designs, and therapeutic drugs. In the foreseeable future, the continuing impacts of COVID-19 are inevitable. Anti-viral medications against SARS-CoV-2 are the key to tackling the pandemic.

New prescription medicine development is a decade-long and expensive (over $\$ 1$ billion) process that consists of preclinical research, clinical trials, and commercialization (Hughes et al., 2011). Facing such challenging work, bioactive molecules of natural origin have been proven to be an excellent source for drug discovery, especially for cancer, infectious diseases, cardiovascular diseases, and multiple sclerosis (Atanasov et al., 2021). Their wide range of pharmacological activities include anti-cancer, anti-bacterial, anti-viral, anti-malaria, antiinflammatory, anti-oxidation, anti-ageing, anti-hypertension, anti-diabetic, and immune regulation activities ( $\mathrm{Tu}, 2011$; Adnan et al., 2018a; Adnan et al., 2018b; Patel et al., 2019;
Adnan et al., 2020; Mandadi et al., 2020; Patel et al., 2020; Siddiqui et al., 2020). In addition, the synergy of natural bioactive molecules with conventional drugs has been widely demonstrated and applied in clinical treatments (Lee et al., 2008; Sung and Lee, 2008; Russo et al., 2017). Therefore, it is a feasible strategy to identify and screen natural bioactive molecules as therapeutic agents that can effectively treat COVID-19. This review will summarize the viral biological characteristics and invasion mechanisms and highlight potential drug targets for the treatment of COVID-19. More significantly, the listed natural products with anti-CoV properties will be arranged by their different targets in the viral life cycle, mainly focusing on natural bioactive molecules with clear targets and activity data.

\section{VIRAL BIOLOGICAL CHARACTERISTICS}

\section{Genome Organization}

As a novel $\beta$-coronavirus, the first SARS-CoV-2 genome sequence (NC_045512.2) was immediately reported in the early stage of the outbreak, which is closely related to BatCoV RaTG13 (about 96.3\%), SARS-CoV (about 79\%), and MERS-CoV (about 50\%) (Lu et al., 2020; Paraskevis et al., 2020; Wu et al., 2020). Its 29.9 kb genome encodes as many as 14 open reading frames (ORFs), including five functional ORFs and nine putative accessory 
factors (Figure 1B). From $5^{\prime}$ to $3^{\prime}$, ORF1a and ORF1b occupy two-thirds of the whole genome and encode two polyproteins which are auto-proteolytically processed into 16 non-structural proteins (Nsp1-16). Then, the genome encodes four structural proteins-spike (S), envelope (E), membrane (M), and nucleocapsid $(\mathrm{N})$-with nine putative accessory factors encoded between them. Compared with SARS-CoV, the genome organization of SARS-CoV-2 shows few differences in the ORFs and Nsps. The main differences between the two are concentrated in just ORF3b, ORF8, ORF10, and spike (Chan et al., 2020; Chellapandi and Saranya, 2020; Gordon et al., 2020; Hu et al., 2020).

\section{Genomic Products} Non-structural Gene Products

Viruses express their genome products by hijacking the host's translation machinery. The large ORF1a/b are initially translated into polyproteins (ppla, pplab) and then auto-proteolytically processed into 16 non-structural proteins (Nsps) that possess specific and essential roles in the viral life cycle. Due to their almost identical sequences in many of the genomic products, the functions and roles of gene products of SARS-CoV-2 are predicted with confidence based on previous extensive studies on those of SARS-CoV. Nsp1 is predicted to be a host translation inhibitor that forms interaction with the $40 \mathrm{~S}$ ribosomes of the host and induces host mRNA degradation (Kamitani et al., 2006; Narayanan et al., 2008; Tohya et al., 2009; Huang et al., 2011). Nsp3, known as papain-like protease $\left(\mathrm{PL}^{\mathrm{pro}}\right)$, is the largest multidomain protein produced by CoVs and acts as a scaffold protein to interact with itself and to bind to other viral Nsps or host proteins; for example, Nsp3, Nsp4, and Nsp6 form a complex and are involved in viral replication (von Brunn et al., 2007; Imbert et al., 2008; Pfefferle et al., 2011; Chen et al., 2014; Ma-Lauer et al., 2016; Lei et al., 2018). Nsp5, also named main protease $\left(\mathrm{M}^{\mathrm{pro}}\right)$ or $3 \mathrm{C}$-like protease $\left(3 \mathrm{CL}^{\mathrm{pro}}\right)$, is a cysteine protease that can cleave the polyproteins at 11 sites and plays a vital role for the viral replication (Chou et al., 2003; Yin et al., 2007; Pfefferle et al., 2011; Chellapandi and Saranya, 2020). Interestingly, Nsp3 and Nsp5 divide the important work to complete the cleavage of the polyproteins: the former cleaves Nsp1-Nsp3, while the latter cleaves Nsp4-Nsp16 (Anand et al., 2003; Stadler et al., 2003; Prentice et al., 2004). Hence, these two proteases are considered as important targets for the design and development of anti-CoV drugs. Nsp7-Nsp8 complex acts as a primase which assists Nsp12, the viral RNA-dependent RNA polymerase (RdRp), to complete RNA synthesis, and Nsp12, as a core enzyme for the viral RNA replication, is another popular drug target against CoVs (Imbert et al., 2006; te Velthuis et al., 2010; te Velthuis et al., 2012; Xiao et al., 2012; Kirchdoerfer and Ward, 2019). Nsp13, known as NTPase/helicase, is an enzyme of the SF1 family with NTP hydrolysis activity and is translocated along with the nucleic acids by hydrolyzing ATP to retain both dsRNA and dsDNA unwinding activities; it is also considered as an attractive target for anti-CoVs (Seybert et al., 2000; Tanner et al., 2003; Ivanov et al., 2004b; Lee et al., 2010; Adedeji et al., 2012). Nsp10, a critical co-factor for activation of multiple replicative enzymes, is known to interact with both Nsp14 and Nsp16, stimulating their respective $3^{\prime}-5^{\prime}$ exoribonuclease (ExoN) and 2'-Omethyltransferase activities (Decroly et al., 2008; Lugari et al., 2010; Decroly et al., 2011; Bouvet et al., 2012; Bouvet et al., 2014). In addition to the $\mathrm{N}$-terminal ExoN function, the $\mathrm{C}$-terminal of Nsp14 serves as N7-methyltransferase (N7Tase) (Chen et al., 2009). Nsp15, known as uridylate-specific endoribonuclease (NendoU), cooperates with Nsp14 to finish the precise cleavage of the viral RNA genome (Ivanov et al., 2004a; Bhardwaj et al., 2006; Fehr and Perlman, 2015; Xu et al., 2020).

\section{Structural Gene Products}

Four structural proteins, spike (S), envelope (E), membrane (M), and nucleocapsid $(\mathrm{N})$, are expressed in host cells and play crucial roles in the viral infestation, assembly, and release. The $S$ protein of SARS-CoV-2, which contains an N-terminal S1 subunit (residue 14-685) and a C-terminal S2 region (residue 686-1273), is essential for the viral infestation by binding to the same cell surface receptor of SARS-CoV, angiotensinconverting enzyme 2 (ACE2) (Hoffmann et al., 2020; Ou et al., 2020). The S1 subunit contains a receptor-binding domain (RBD), which can bind to the peptidase domain (PD) of ACE2, and shares around 70\% identity with SARS-CoV. On the other hand, the S2 subunit, which helps the viral envelop fuse with the cellular membranes, shares $99 \%$ identity with SARS-CoV (Chan et al., 2020; Chellapandi and Saranya, 2020; Hu et al., 2020; Wrapp et al., 2020). Due to the essential role in viral infestation, targeting the $\mathrm{S}$ protein is a promising strategy for developing a drug to fight against SARS-CoV-2 (Xia et al., 2019). The small E protein plays an essential role in virus assembly and release and is implicated in the induction of host apoptosis (Liu et al., 2007; Ruch and Machamer, 2012; Schoeman and Fielding, 2019). The $\mathrm{M}$ protein, which is the most abundant viral constituent and acts as a scaffold protein, controls the assembly of viral particles and ensures the correct morphology of the virion (Arndt et al., 2010; Siu et al., 2014; Fung and Liu, 2019). The N protein forms the viral nucleocapsid with the RNA genome and participates in the viral RNA synthesis (Hatakeyama et al., 2008; Chang et al., 2014; McBride et al., 2014).

\section{Other Gene Products}

Beyond the functional proteins, the viral genome controls the expression of nine accessory proteins, which are usually regarded as dispensable for replication or structure but play other not entirely clear roles in the viral life cycle. For example, product of ORF3a is the largest accessory protein to be efficiently expressed on the cell surface and acts as an ion channel that may promote virus release (Lu et al., 2006; Michel et al., 2020). Several studies have shown that various ORF3b proteins of bat SARS-related$\mathrm{CoV}$ strains have different interferon antagonistic activities. However, ORF3b of SARS-CoV-2 encodes a novel protein with no homology to ORF3b of SARS-CoV, whose function has yet to be investigated (Kopecky-Bromberg et al., 2007; Zhou et al., 2012; Chan et al., 2020). ORF8 of SARS-CoV is one of the most rapidly evolving regions among SARS-CoV genomes and is related to the viral adaptation to humans following interspecies transmission and replication, while ORF8 of SARS-CoV-2 is distant from that of known CoVs 


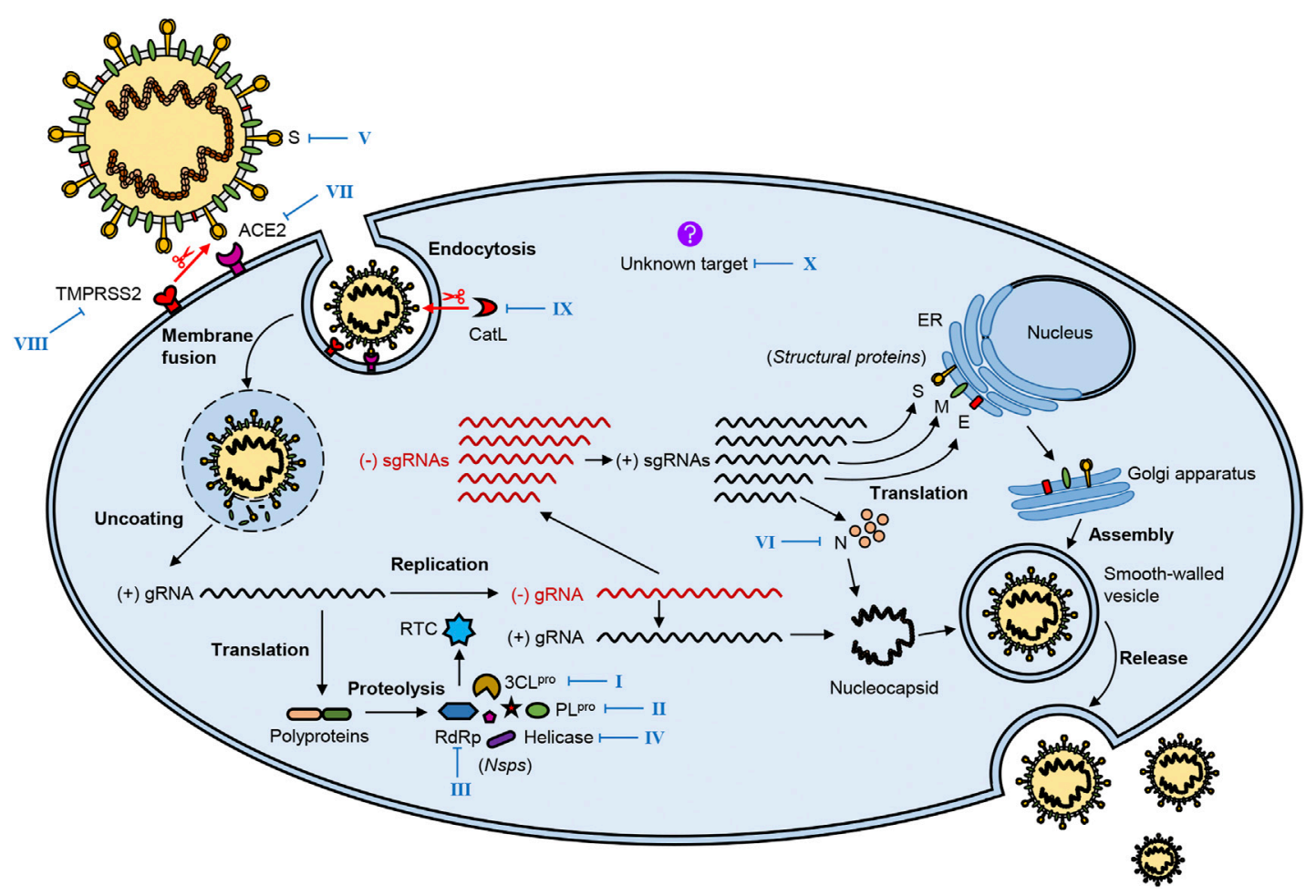

FIGURE 2 | The life cycle of SARS-CoV-2 and potential therapeutic targets. SARS-CoV-2 infestation of host cells undergoes the following processes: virus attachment and entry, genome replication and transcription, virion assembly, and release. Several vital viral proteins, including $3 \mathrm{CL}^{\text {pro }}$, $\mathrm{PL}^{\text {pro }}$, RdRp, helicase, spike (S), and nucleocapsid $(\mathrm{N})$, play essential roles in the life cycle of SARS-CoV-2, which may be potential therapeutic targets. Meanwhile, key host proteins significantly contribute to viral infection, such as receptor ACE2, proteases TMPRSS2, and CatL. The blue Roman numbers in the figure refer to natural bioactive molecules that inhibit different potential targets in Figures 3-8 and Tables 1-3.

(Ceraolo and Giorgi, 2020; Chan et al., 2020; Michel et al., 2020). Overall, accessory proteins have not been adequately studied due to their dispensable roles in viral replication or structure and the fact that ORFs are short and overlapping, posing a challenge for bioinformatic prediction. However, further studies of these accessory proteins may reveal the promise of these proteins in the diagnosis, treatment, and prevention of coronaviruses because of their unique roles.

\section{VIRAL INVASION MECHANISMS}

After SARS-CoV-2 enters the human body, it infects the host cells mainly through these processes: virus attachment and entry, and genome replication and transcription, as well as virion assembly and release (Figure 2). These processes are accomplished through the interaction of the virus and the host cell.

\section{Viral Attachment and Entry}

Proteolytic activation of the $S$ protein plays a crucial role in SARSCoV-2 attachment to and entry into host cells. The following steps complete this process: 1) recognition of the $S$ protein and binding it to the cellular receptor; 2) alteration of the conformation and proteolysis of the S protein; 3) activation of fusion of the virion and endocytosis (Pillay, 2020).
The first step, also regarded as the beginning of SARS-CoV-2 life cycle, is the interaction of the $S$ protein with the cell surface receptor ACE2, in which the $\mathrm{RBD}$ located at the $\mathrm{S} 1$ subunit binds to the carboxypeptidase domain of ACE2. Then, this interaction triggers a dramatic conformational change in the S2 subunit, leading to exposure and cleavage of the cleavage site at the S2 subunit which can be processed by the host cellular proteases such as cell surface transmembrane protease serine 2 (TMPRSS2) (Hoffmann et al., 2020; Matsuyama et al., 2020; Ou et al., 2020). After proteolysis of the $\mathrm{S}$ protein, the virion begins to fuse with the host cell membrane and enter the host cell through endocytosis.

The cleavage of the S protein is significant for SARS-CoV-2 infection and can occur at two cleavage sites processed by different proteases. The first cleavage site located at the S2 subunit can be targeted by the host cellular proteases such as TMPRSS2, which has a crucial role in activating membrane fusion between the virus and the host cell. Similarly, TMPRSS4, another serine protease in the same family, plays a similar role to TMPRSS2 in SARS-CoV-2 infection. Furthermore, recent studies have shown that camostat mesylate, a selective inhibitor of TMPRSS, can inhibit SARS-CoV-2 infection (Hoffmann et al., 2020; Zang et al., 2020). In addition, some other host cell proteases, such as cathepsin L (CatL), can also proteolytically activate the $\mathrm{S}$ protein of SARS-CoV-2 and then 
initiate the process of cellular entry (Ou et al., 2020). The second cleavage site is the furin cleavage site (Arg-Arg-Ala-Arg) between the S1 and S2 domains, common to other human CoVs like MERS-CoV but interestingly absent from SARS-CoV. The furin cleavage site can reduce the stability of the $S$ protein and facilitate the conformational change required for RBD exposure and the subsequent binding to ACE2. Furin-like proteases are widely expressed in various cell types, especially in the respiratory tract, so the presence of the furin cleavage site in the $S$ protein is thought to increase the infectivity of SARS-CoV-2 or alter its pathogenicity (Walls et al., 2020; Wrobel et al., 2020).

\section{Genome Replication and Transcription}

After the completion of virus attachment and entry, the nucleocapsid is released into the host cytoplasm; then, virus replication is initiated in the cytoplasm. The virus hijacks the ribosome of the host cell; this is followed by the translation and auto-proteolysis of the polyproteins ppla and pplab into 16 Nsps, which altogether form the replicase-transcriptase complex (RTC) that controls the processes of replication and translation. Mediated by RTC, the viral genomic RNA is replicated to fulllength negative-sense (-)RNA; then, the (-)RNA is used as a template to synthesize new genomic (+)RNA and a series of different sgRNAs, the latter of which are translated into viral structural and accessory proteins (Ziebuhr, 2005; Masters, 2006).

\section{Virion Assembly and Release}

When the base components are prepared, the virion assembly follows. First, the membrane-bound structural proteins, E, M, and $S$, are inserted into the endoplasmic reticulum (ER) and then transported to the ER-Golgi intermediate compartment (ERGIC). The $\mathrm{N}$ protein wraps the new genomic RNA to form a nucleocapsid, which then transits to ERGIC. The nucleocapsid and membrane-bound components coalesce to assemble virion mediated by the M protein in ERGIC. Finally, progeny virions are transported to the plasma membrane in smooth-walled vesicles and released by exocytosis (Masters, 2006; Fung and Liu, 2019; Russo et al., 2020).

\section{ANTI-COV BIOACTIVE MOLECULES TARGETING DIFFERENT PROTEINS}

In the previous sections, we have described fundamental biological information of SARS-CoV-2, the vast majority of which is conserved among other known coronaviruses especially SARS-CoV. In this dire SARS-CoV-2 pandemic with no effective drug, screening natural bioactive molecules from natural products with known anti-CoV activity can significantly accelerate the development of effective drugs against SARS-CoV2. In this section, we will summarize natural bioactive molecules that have been reported to exhibit anti-CoV activity targeting different vital proteins, including several crucial viral and host proteins. As natural agents against SARS-CoV are the most widely reported, we will mainly focus on natural bioactive molecules found in SARS-CoV studies and introduce a few natural inhibitors against MERS-CoV or SARS-CoV-2. The description of these natural bioactive molecules will be developed according to their different targets.

\section{Viral Proteins \\ Viral Proteases}

During the replication of the virus, $\mathrm{PL}^{\text {pro }}$ and $3 \mathrm{CL}^{\text {pro }}$ are responsible for the cleavage of the polyproteins; as a result, they are considered as the most popular targets for the design and development of anti-CoV drugs. Many synthetic compounds targeting these proteases have been reported, such as rupintrivir, lopinavir, and ritonavir. Due to the inherent peptidase activity, a lot of work has been done in designing peptidomimetic inhibitors for these proteases, which will not be discussed here in detail ( $\mathrm{Hu}$ et al., 2020; Christy et al., 2021). Furthermore, many natural bioactive molecules, mostly flavonoids, have also been shown to inhibit $\mathrm{PL}^{\text {pro }}$ and $3 \mathrm{CL}^{\text {pro }}$ (Figure 3; Table 1).

Among the natural products studied for their activity against SARS-CoV, the largest number of bioactive molecules has been reported to have $3 \mathrm{CL}^{\text {pro }}$ inhibitory activity (I, Figure 3A). Lin et al. used cell-free and cell-based cleavage assays to study antiSARS-CoV $3 \mathrm{CL}^{\text {pro }}$ activities of Isatis tinctoria L. root extract, five major compounds of Isatis tinctoria L. root, and seven plantderived phenolic compounds. Their study showed that Isatis tinctoria L. root extract (1), indigo (2), sinigrin (3), aloe emodin (4), and hesperetin (5) exhibited significant inhibitory activity against SARS-CoV $3 \mathrm{CL}^{\text {pro }}$ in the micromolar range. In particular, hesperetin showed the best activity among these compounds and dose-dependently inhibited cleavage activity

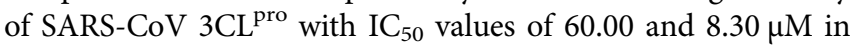
cell-free and cell-based cleavage assays, respectively (Lin et al., 2005). Interestingly, although quercetin was reported to have anti-SARS-CoV activity, it did not show anti-3CL ${ }^{\text {pro }}$ activity in this study (Yi et al., 2004; Lin et al., 2005). However, in several subsequent studies, quercetin showed inhibitory activity against SARS-CoV $3 \mathrm{CL}^{\text {pro }}$ or was used as a positive control. A natural glycoside derivative of quercetin, quercetin-3- $\beta$-galactoside (6), was shown to block the cleavage activity of SARS-CoV 3CL $\mathrm{CL}^{\text {pro }}$ with an $\mathrm{IC}_{50}$ of $42.76 \mu \mathrm{M}$. Through molecular modeling and Q189A mutation of $3 \mathrm{CL}^{\text {pro }}$, Gln189 was identified as an important amino acid residue that played a vital role in quercetin-3- $\beta$-galactoside binding to $3 \mathrm{CL}^{\text {pro }}$. The $\mathrm{Q} 186 \mathrm{~A}$ mutation did not change the enzymatic activity of $3 \mathrm{CL}^{\text {pro, }}$, while the SPR and FRET assay results showed that both the binding affinity and the inhibitory potency of quercetin-3$\beta$-galactoside to the mutated $3 \mathrm{CL}^{\text {pro }}$ were significantly lower than those to the wild-type $3 \mathrm{CL}^{\text {pro }}$ (Chen et al., 2006). Ryu et al. implemented FRET assay to evaluate the anti-SARS-CoV $3 \mathrm{CL}^{\text {pro }}$ activity of 12 compounds extracted from Torreya nucifera (L.) Siebold \& Zucc., including eight diterpenoids and four biflavonoids, and abietic acid $\left(15, \mathrm{IC}_{50}=58.00 \mu \mathrm{M}\right)$, apigenin (20, $\left.\mathrm{IC}_{50}=280.80 \mu \mathrm{M}\right)$, luteolin $\left(21, \mathrm{IC}_{50}=20.20 \mu \mathrm{M}\right)$, and quercetin $\left(22, \mathrm{IC}_{50}=23.80 \mu \mathrm{M}\right)$ were used as positive control compounds. Among these 12 compounds, the biflavone amentoflavone (16) showed the most potent $3 \mathrm{CL}^{\text {pro }}$ inhibitory effect with an $\mathrm{IC}_{50}$ of $8.30 \mu \mathrm{M}$ (Ryu et al., 2010a). In another study, the anti-3CL ${ }^{\text {pro }}$ activities of seven flavonoid compounds were evaluated by in vitro $3 \mathrm{CL}^{\text {pro }}$ inhibition and kinetic assays, 
Chen et al.

Natural Bioactive Molecules Against SARS-CoV-2

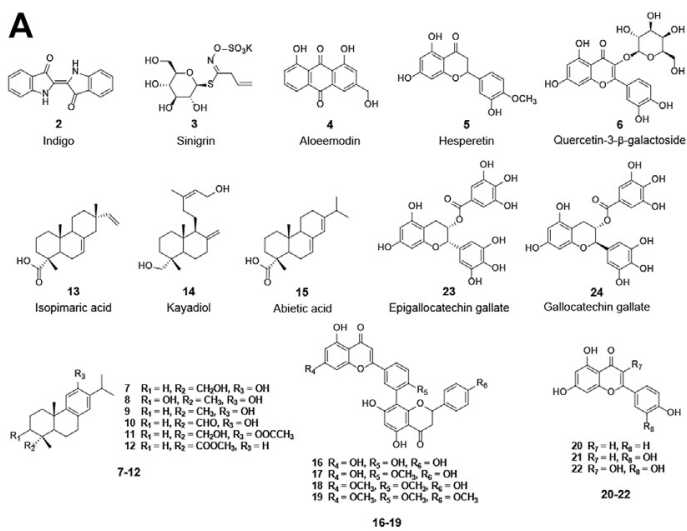

B
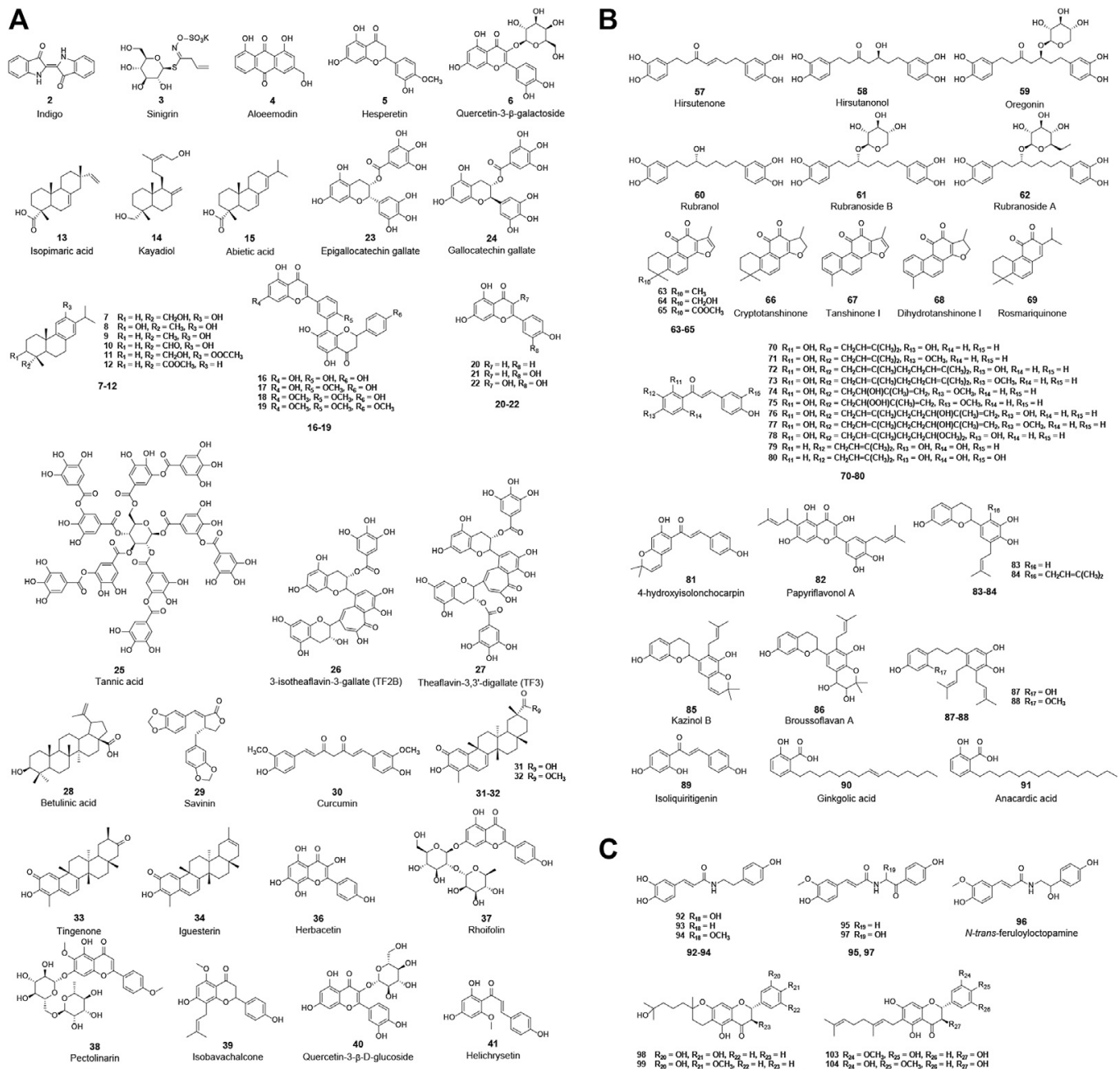

C
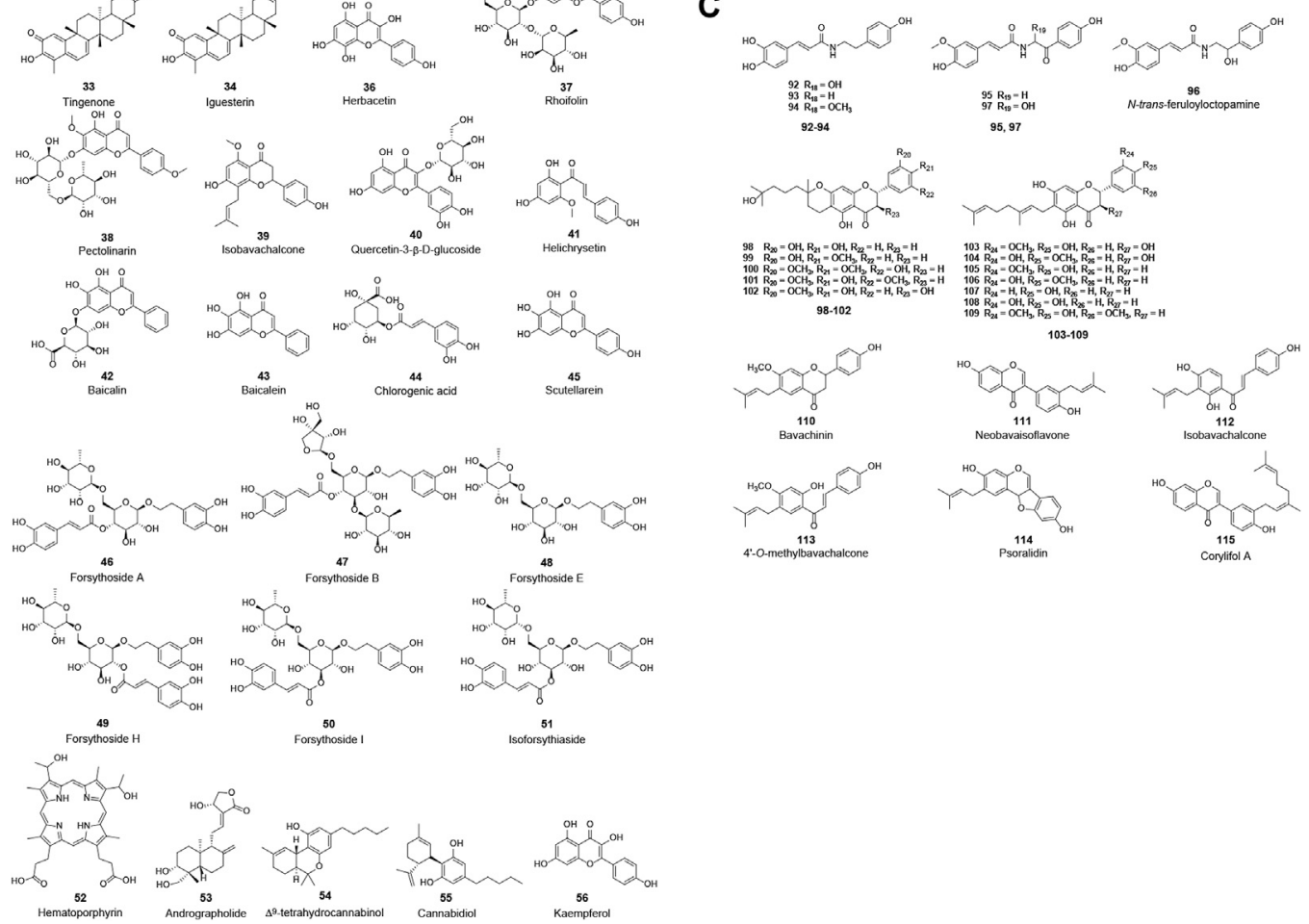

50
Forsythoside

51
Isoforsythiaside

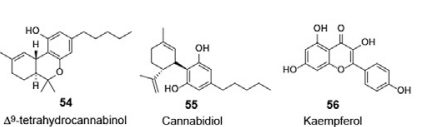

FIGURE 3 | Chemical structure of different natural compounds targeting (A) $3 C L^{\text {pro }}(\mathbf{I})$, (B) both $3 C L^{\text {pro }}$ and $P L^{\text {pro }}$, and (C) PL pro $(\mathbf{I I})$. Quercetin-3- $\beta$-galactoside (6), quercetin (22), curcumin (31), and kaempferol (57) inhibit both $3 \mathrm{CL}^{\text {pro }}$ and $\mathrm{PL}^{\text {pro }}$ but are not repeatedly displayed in (B).

Frontiers in Pharmacology | www.frontiersin.org

6

August 2021 | Volume 12 | Article 702472 
TABLE 1 | Natural compounds targeting viral proteins: 3CL pro $(\mathbf{I}), \mathrm{PL}^{\text {pro }}(\mathbf{I I})$, RdRp (III), NTPase/helicase (IV), S protein (V), and N protein (VI).

\begin{tabular}{|c|c|c|c|c|c|}
\hline No & Viral strain & Compound & $\mathrm{IC}_{50} / \mathrm{EC}_{50}$ & Target & References \\
\hline 1 & SARS-CoV & Isatis tinctoria L. root extract & $53.80 \mu \mathrm{g} / \mathrm{ml}$ & $3 C L^{\text {pro }}$ & Lin et al. (2005) \\
\hline 2 & SARS-CoV & Indigo & $300.00 \mu \mathrm{M}$ & $3 C L^{\text {pro }}$ & Lin et al. (2005) \\
\hline 3 & SARS-CoV & Sinigrin & $121.00 \mu \mathrm{M}$ & $3 C L^{\text {pro }}$ & Lin et al. (2005) \\
\hline 4 & SARS-CoV & Aloe emodin & $132.00 \mu \mathrm{M}$ & $3 C L^{\text {pro }}$ & Lin et al. (2005) \\
\hline 5 & SARS-CoV & Hesperetin & $60.00 \mu \mathrm{M}$ & $3 C L^{\text {pro }}$ & Lin et al. (2005) \\
\hline 6 & SARS-CoV & Quercetin-3- $\beta$-galactoside & $42.79 \mu \mathrm{M}$ & $3 C L^{\text {pro }}$ & Chen et al. (2006) \\
\hline 7 & SARS-CoV & 18-Hydroxyferruginol & $45.80 \mu \mathrm{M}$ & $3 C L^{\text {pro }}$ & Ryu et al. (2010a) \\
\hline 8 & SARS-CoV & Honokiol & $39.10 \mu \mathrm{M}$ & $3 C L^{\text {pro }}$ & Ryu et al. (2010a) \\
\hline 9 & SARS-CoV & Ferruginol & $92.70 \mu \mathrm{M}$ & $3 \mathrm{CL}^{\text {pro }}$ & Ryu et al. (2010a) \\
\hline 10 & SARS-CoV & 18-Oxoferruginol & $70.50 \mu \mathrm{M}$ & $3 C L^{\text {pro }}$ & Ryu et al. (2010a) \\
\hline 11 & SARS-CoV & O-acetyl-18-hydroxyferruginol & $78.60 \mu \mathrm{M}$ & $3 C L^{\text {pro }}$ & Ryu et al. (2010a) \\
\hline 12 & SARS-CoV & Methyl dehydroabietate & $46.70 \mu \mathrm{M}$ & $3 C L^{\text {pro }}$ & Ryu et al. (2010a) \\
\hline 13 & SARS-CoV & Isopimaric acid & $28.90 \mu \mathrm{M}$ & $3 C L^{\text {pro }}$ & Ryu et al. (2010a) \\
\hline 14 & SARS-CoV & Kayadiol & $75.20 \mu \mathrm{M}$ & $3 C L^{\text {pro }}$ & Ryu et al. (2010a) \\
\hline 15 & SARS-CoV & Abietic acid & $58.00 \mu \mathrm{M}$ & $3 C L^{\text {pro }}$ & Ryu et al. (2010a) \\
\hline 16 & SARS-CoV & Amentoflavone & $8.30 \mu \mathrm{M}$ & $3 C L^{\text {pro }}$ & Ryu et al. (2010a) \\
\hline 17 & SARS-CoV & Bilobetin & $72.30 \mu \mathrm{M}$ & $3 C L^{\text {pro }}$ & Ryu et al. (2010a) \\
\hline 18 & SARS-CoV & Ginkgetin & $32.00 \mu \mathrm{M}$ & $3 C L^{\text {pro }}$ & Ryu et al. (2010a) \\
\hline 19 & SARS-CoV & Sciadopitysin & $38.40 \mu \mathrm{M}$ & $3 C L^{\text {pro }}$ & Ryu et al. (2010a) \\
\hline 20 & SARS-CoV & Apigenin & $280.80 \mu \mathrm{M}$ & $3 C L^{\text {pro }}$ & Ryu et al. (2010a) \\
\hline 21 & SARS-CoV & Luteolin & $20.20 \mu \mathrm{M}$ & $3 C L^{\text {pro }}$ & Ryu et al. (2010a) \\
\hline 22 & SARS-CoV & Quercetin & $23.80 \mu \mathrm{M}$ & $3 C L^{\text {pro }}$ & Ryu et al. (2010a) \\
\hline 22 & SARS-CoV & Quercetin & $73.00 \mu \mathrm{M}$ & $3 \mathrm{CL}^{\text {pro }}$ & Nguyen et al. (2012) \\
\hline 23 & SARS-CoV & Epigallocatechin gallate & $73.00 \mu \mathrm{M}$ & $3 \mathrm{CL}^{\text {pro }}$ & Nguyen et al. (2012) \\
\hline 24 & SARS-CoV & Gallocatechin gallate & $47.00 \mu \mathrm{M}$ & $3 C L^{\text {pro }}$ & Nguyen et al. (2012) \\
\hline 25 & SARS-CoV & Tannic acid & $3.00 \mu \mathrm{M}$ & $3 \mathrm{CL}^{\text {pro }}$ & Chen et al. (2005) \\
\hline 26 & SARS-CoV & 3-Isotheaflavin-3-gallate (TF2B) & $7.00 \mu \mathrm{M}$ & $3 C L^{\text {pro }}$ & Chen et al. (2005) \\
\hline 27 & SARS-CoV & Theaflavin-3,3'-digallate (TF3) & $9.50 \mu \mathrm{M}$ & $3 C L^{\text {pro }}$ & Chen et al. (2005) \\
\hline 28 & SARS-CoV & Betulinic acid & $10.00 \mu \mathrm{M}$ & $3 C L^{\text {pro }}$ & Wen et al. (2007) \\
\hline 29 & SARS-CoV & Savinin & $25.00 \mu \mathrm{M}$ & $3 C L^{\text {pro }}$ & Wen et al. (2007) \\
\hline 30 & SARS-CoV & Curcumin & $40.00 \mu \mathrm{M}$ & $3 C L^{\text {pro }}$ & Wen et al. (2007) \\
\hline 30 & SARS-CoV & Curcumin & $23.50 \mu \mathrm{M}$ & $3 C L^{\text {pro }}$ & Ryu et al. (2010b) \\
\hline 31 & SARS-CoV & Celastrol & $10.30 \mu \mathrm{M}$ & $3 C L^{\text {pro }}$ & Ryu et al. (2010b) \\
\hline 32 & SARS-CoV & Pristimererin & $5.50 \mu \mathrm{M}$ & $3 C L^{\text {pro }}$ & Ryu et al. (2010b) \\
\hline 33 & SARS-CoV & Tingenone & $9.90 \mu \mathrm{M}$ & $3 C L^{\text {pro }}$ & Ryu et al. (2010b) \\
\hline 34 & SARS-CoV & Iguesterin & $2.60 \mu \mathrm{M}$ & $3 C L^{\text {pro }}$ & Ryu et al. (2010b) \\
\hline 35 & SARS-CoV & Rheum palmatum L. extract $(\mathrm{RH} 121)$ & $13.76 \mu \mathrm{g} / \mathrm{ml}$ & $3 C L^{\text {pro }}$ & Luo et al. (2009) \\
\hline 36 & SARS-CoV & Herbacetin & $33.17 \mu \mathrm{M}$ & $3 C L^{\text {pro }}$ & Jo et al. (2020) \\
\hline 37 & SARS-CoV & Rhoifolin & $27.45 \mu \mathrm{M}$ & $3 C L^{\text {pro }}$ & Jo et al. (2020) \\
\hline 38 & SARS-CoV & Pectolinarin & $37.78 \mu \mathrm{M}$ & $3 C L^{\text {pro }}$ & Jo et al. (2020) \\
\hline 36 & MERS-CoV & Herbacetin & $40.59 \mu \mathrm{M}$ & $3 C L^{\text {pro }}$ & Jo et al. (2019) \\
\hline 39 & MERS-CoV & Isobavachalcone & $35.85 \mu \mathrm{M}$ & $3 C L^{\text {pro }}$ & Jo et al. (2019) \\
\hline 40 & MERS-CoV & Quercetin-3- $\beta$-D-glucoside & $37.03 \mu \mathrm{M}$ & $3 C L^{\text {pro }}$ & Jo et al. (2019) \\
\hline 41 & MERS-CoV & Helichrysetin & $67.04 \mu \mathrm{M}$ & $3 C L^{\text {pro }}$ & Jo et al. (2019) \\
\hline 22 & SARS-CoV-2 & Quercetin & $\mathrm{K}_{\mathrm{i}}=7.00 \mu \mathrm{M}$ & $3 C L^{\text {pro }}$ & Abian et al. (2020) \\
\hline 42 & SARS-CoV-2 & Baicalin & $6.41 \mu \mathrm{M}$ & $3 C L^{\text {pro }}$ & Su et al. (2020) \\
\hline 43 & SARS-CoV-2 & Baicalein & $0.94 \mu \mathrm{M}$ & $3 C L^{\text {pro }}$ & Su et al. (2020) \\
\hline 44 & SARS-CoV-2 & Chlorogenic acid & $39.48 \mu \mathrm{M}$ & $3 C L^{\text {pro }}$ & Su et al. (2020) \\
\hline 45 & SARS-CoV-2 & Scutellarein & $3.02 \mu \mathrm{M}$ & $3 C L^{\text {pro }}$ & Su et al. (2020) \\
\hline 46 & SARS-CoV-2 & Forsythoside A & $3.18 \mu \mathrm{M}$ & $3 C L^{\text {pro }}$ & Su et al. (2020) \\
\hline 47 & SARS-CoV-2 & Forsythoside B & $2.88 \mu \mathrm{M}$ & $3 C L^{\text {pro }}$ & Su et al. (2020) \\
\hline 48 & SARS-CoV-2 & Forsythoside E & $6.88 \mu \mathrm{M}$ & $3 C L^{\text {pro }}$ & Su et al. (2020) \\
\hline 49 & SARS-CoV-2 & Forsythoside $\mathrm{H}$ & $10.17 \mu \mathrm{M}$ & $3 C L^{\text {pro }}$ & Su et al. (2020) \\
\hline 50 & SARS-CoV-2 & Forsythoside I & $5.47 \mu \mathrm{M}$ & $3 C L^{\text {pro }}$ & Su et al. (2020) \\
\hline 51 & SARS-CoV-2 & Isoforsythiaside & $5.85 \mu \mathrm{M}$ & $3 C L^{\text {pro }}$ & Su et al. (2020) \\
\hline 25 & SARS-CoV-2 & Tannic acid & $13.40 \mu \mathrm{M}$ & $3 C L^{\text {pro }}$ & Wang et al. (2020) \\
\hline 25 & SARS-CoV-2 & Tannic acid & $2.10 \mu \mathrm{M}$ & $3 C L^{\text {pro }}$ & Coelho et al. (2020) \\
\hline 52 & SARS-CoV-2 & Hematoporphyrin & $3.90 \mu \mathrm{M}$ & $3 C L^{\text {pro }}$ & Coelho et al. (2020) \\
\hline 53 & SARS-CoV-2 & Andrographolide & $15.05 \mu \mathrm{M}$ & $3 \mathrm{CL}^{\text {pro }}$ & Shi et al. (2020) \\
\hline 54 & SARS-CoV-2 & $\Delta^{9}$-Tetrahydrocannabinol & $10.25 \mu \mathrm{M}$ (antiviral activity) & $3 C L^{\text {pro }}$ & Raj et al. (2021) \\
\hline 55 & SARS-CoV-2 & Cannabidiol & $7.91 \mu \mathrm{M}$ (antiviral activity) & $3 C L^{\text {pro }}$ & Raj et al. (2021) \\
\hline 56 & SARS-CoV-2 & Kaempferol & $34.46 \mu \mathrm{M}$ (antiviral activity) & $3 C L^{\text {pro }}$ & Khan et al. (2021) \\
\hline 57 & SARS-CoV & Hirsutenone & $\begin{array}{c}36.20 \mu \mathrm{M} \\
4.10 \mu \mathrm{M}\end{array}$ & $\begin{array}{l}3 C L^{\text {pro }} \\
P L^{\text {pro }}\end{array}$ & Park et al. (2012b) \\
\hline
\end{tabular}


TABLE 1 | (Continued) Natural compounds targeting viral proteins: $3 \mathrm{CL}^{\text {pro }}$ (I), PL ${ }^{\text {pro }}$ (II), RdRp (III), NTPase/helicase (IV), S protein (V), and N protein (VI).

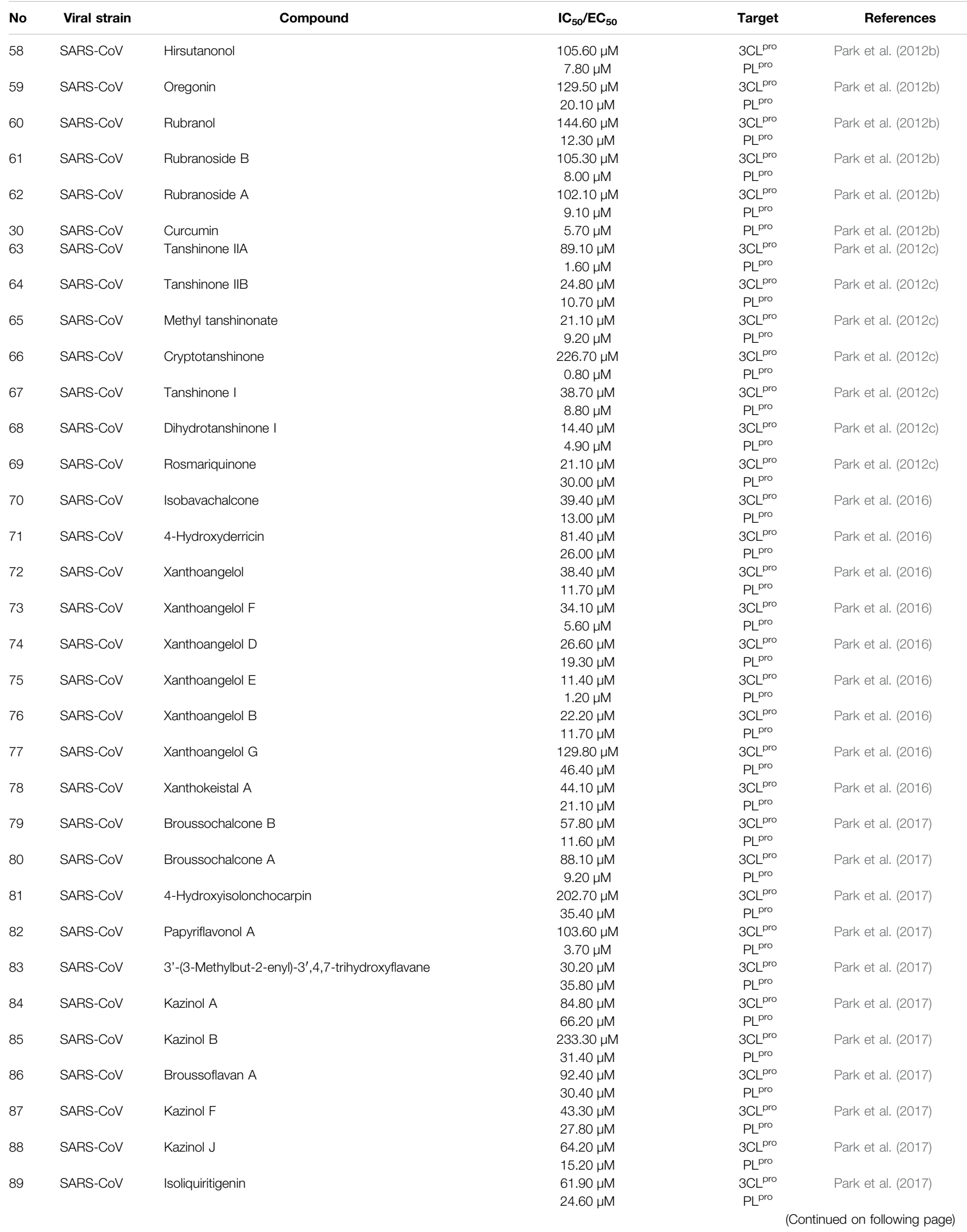


TABLE 1 | (Continued) Natural compounds targeting viral proteins: $3 \mathrm{CL}^{\text {pro }}(\mathbf{I}), \mathrm{PL}^{\text {pro }}$ (II), RdRp (III), NTPase/helicase (IV), S protein (V), and N protein (VI).

\begin{tabular}{|c|c|c|c|c|c|}
\hline No & Viral strain & Compound & $\mathrm{IC}_{50} / \mathrm{EC}_{50}$ & Target & References \\
\hline 56 & SARS-CoV & Kaempferol & $\begin{array}{c}116.30 \mu \mathrm{M} \\
16.30 \mu \mathrm{M}\end{array}$ & $\begin{array}{l}3 C L^{\text {pro }} \\
\mathrm{PL}^{\text {pro }}\end{array}$ & Park et al. (2017) \\
\hline 22 & SARS-CoV & Quercetin & $\begin{array}{c}52.70 \mu \mathrm{M} \\
8.60 \mu \mathrm{M}\end{array}$ & $\begin{array}{l}3 \mathrm{CL}^{\text {pro }} \\
\mathrm{PL}^{\text {pro }}\end{array}$ & Park et al. (2017) \\
\hline 6 & SARS-CoV & Quercetin-3- $\beta$-galactoside & $\begin{array}{c}128.80 \mu \mathrm{M} \\
51.90 \mu \mathrm{M}\end{array}$ & $\begin{array}{l}3 \mathrm{CL}^{\text {pro }} \\
\mathrm{PL}^{\text {pro }}\end{array}$ & Park et al. (2017) \\
\hline 90 & SARS-CoV-2 & Ginkgolic acid & $\begin{array}{c}1.79 \mu \mathrm{M} \\
16.30 \mu \mathrm{M}\end{array}$ & $\begin{array}{l}\text { 3CL pro } \\
\mathrm{PL}^{\text {pro }}\end{array}$ & Chen et al. (2021) \\
\hline 91 & SARS-CoV-2 & Anacardic acid & $\begin{array}{c}2.07 \mu \mathrm{M} \\
17.08 \mu \mathrm{M}\end{array}$ & $\begin{array}{l}\text { 3CL pro } \\
\mathrm{PL}^{\text {pro }}\end{array}$ & Chen et al. (2021) \\
\hline 92 & SARS-CoV & $N$-trans-caffeoyltyramine & $44.40 \mu \mathrm{M}$ & $P^{\text {pro }}$ & Song et al. (2014) \\
\hline 93 & SARS-CoV & $\mathrm{N}$-trans-coumaroyltyramine & $38.80 \mu \mathrm{M}$ & $P L^{\text {pro }}$ & Song et al. (2014) \\
\hline 94 & SARS-CoV & $N$-trans-feruloyltyramine & $70.10 \mu \mathrm{M}$ & $P L^{\text {pro }}$ & Song et al. (2014) \\
\hline 95 & SARS-CoV & Terrestriamide & $21.50 \mu \mathrm{M}$ & $P L^{\text {pro }}$ & Song et al. (2014) \\
\hline 96 & SARS-CoV & $N$-trans-feruloyloctopamine & $26.60 \mu \mathrm{M}$ & $P L^{\text {pro }}$ & Song et al. (2014) \\
\hline 97 & SARS-CoV & Terrestrimine & $15.80 \mu \mathrm{M}$ & $P L^{\text {pro }}$ & Song et al. (2014) \\
\hline 98 & SARS-CoV & Tomentin A & $6.20 \mu \mathrm{M}$ & $P L^{\text {pro }}$ & Cho et al. (2013) \\
\hline 99 & SARS-CoV & Tomentin B & $6.10 \mu \mathrm{M}$ & $P L^{\text {pro }}$ & Cho et al. (2013) \\
\hline 100 & SARS-CoV & Tomentin C & $11.60 \mu \mathrm{M}$ & $P L^{\text {pro }}$ & Cho et al. (2013) \\
\hline 101 & SARS-CoV & Tomentin D & $12.50 \mu \mathrm{M}$ & $P L^{\text {pro }}$ & Cho et al. (2013) \\
\hline 102 & SARS-CoV & Tomentin E & $5.00 \mu \mathrm{M}$ & $P L^{\text {pro }}$ & Cho et al. (2013) \\
\hline 103 & SARS-CoV & 3'-O-methyldiplacol & $9.50 \mu \mathrm{M}$ & $P L^{\text {pro }}$ & Cho et al. (2013) \\
\hline 104 & SARS-CoV & 4'-O-methyldiplacol & $9.20 \mu \mathrm{M}$ & $P L^{\text {pro }}$ & Cho et al. (2013) \\
\hline 105 & SARS-CoV & $3^{\prime}$-O-methyldiplacone & $13.20 \mu \mathrm{M}$ & $P L^{\text {pro }}$ & Cho et al. (2013) \\
\hline 106 & SARS-COV & 4'-O-methyldiplacone & $12.70 \mu \mathrm{M}$ & $P L$ pro & Cho et al. (2013) \\
\hline 107 & SARS-CoV & Mimulone & $14.40 \mu \mathrm{M}$ & $P L^{\text {pro }}$ & Cho et al. (2013) \\
\hline 108 & SARS-CoV & Diplacone & $10.40 \mu \mathrm{M}$ & $P L^{\text {pro }}$ & Cho et al. (2013) \\
\hline 109 & SARS-CoV & 6-Geranyl-4',5,7-trihydroxy-3',5'-dimethoxyflavanone & $13.90 \mu \mathrm{M}$ & $P L^{\text {pro }}$ & Cho et al. (2013) \\
\hline 110 & SARS-CoV & Bavachinin & $38.40 \mu \mathrm{M}$ & $P L^{\text {pro }}$ & Kim et al. (2014) \\
\hline 111 & SARS-CoV & Neobavaisoflavone & $18.30 \mu \mathrm{M}$ & $P L^{\text {pro }}$ & Kim et al. (2014) \\
\hline 112 & SARS-CoV & Isobavachalcone & $7.30 \mu \mathrm{M}$ & $P L^{\text {pro }}$ & Kim et al. (2014) \\
\hline 113 & SARS-CoV & 4'-O-methylbavachalcone & $10.10 \mu \mathrm{M}$ & $P L$ pro & Kim et al. (2014) \\
\hline 114 & SARS-CoV & Psoralidin & $4.20 \mu \mathrm{M}$ & $P L^{\text {pro }}$ & Kim et al. (2014) \\
\hline 115 & SARS-CoV & Corylifol A & $32.20 \mu \mathrm{M}$ & $P L^{\text {pro }}$ & Kim et al. (2014) \\
\hline 116 & SARS-CoV & Kwan du Bu Fei Dang exact & $471.30 \mu \mathrm{g} / \mathrm{ml}$ & $\mathrm{RdRp}$ & Fung et al. (2011) \\
\hline 117 & SARS-CoV & Houttuynia cordata exact & $251.10 \mu \mathrm{g} / \mathrm{ml}$ & $\mathrm{RdRp}$ & Fung et al. (2011) \\
\hline 118 & SARS-CoV & Ganoderma lucidum exact & $41.90 \mu \mathrm{g} / \mathrm{ml}$ & $\operatorname{RdRp}$ & Fung et al. (2011) \\
\hline 119 & SARS-CoV & Coriolus versicolor exact & $108.40 \mu \mathrm{g} / \mathrm{ml}$ & $\mathrm{RdRp}$ & Fung et al. (2011) \\
\hline 120 & SARS-CoV & Sinomenium acutum exact & $198.60 \mu \mathrm{g} / \mathrm{ml}$ & $\mathrm{RdRp}$ & Fung et al. (2011) \\
\hline 22 & SARS-CoV & Quercetin & $8.10 \mu \mathrm{M}$ & NTPase/helicase & Lee et al. (2009b) \\
\hline 121 & SARS-CoV & Myricetin & $2.71 \mu \mathrm{M}$ & NTPase/helicase & Yu et al. (2012) \\
\hline 122 & SARS-CoV & Scutellarein & $0.86 \mu \mathrm{M}$ & NTPase/helicase & Yu et al. (2012) \\
\hline 123 & SARS-CoV & Tetra-O-galloyl- $\beta$-D-glucose (TGG) & $4.50 \mu \mathrm{M}$ (antiviral activity) & S protein & Yi et al. (2004) \\
\hline 21 & SARS-CoV & Luteolin & $10.60 \mu \mathrm{M}$ (antiviral activity) & S protein & Yi et al. (2004) \\
\hline 124 & SARS-CoV & Emodin & $200.00 \mu \mathrm{M}$ & S protein & Ho et al. (2007) \\
\hline 125 & SARS-CoV & Griffithsin & 48-94 nM (antiviral activity) & S protein & O'Keefe et al. (2010) \\
\hline 126 & SARS-CoV & Urtica dioica L. agglutinin (UDA) & $0.60-2.60 \mu \mathrm{g} / \mathrm{ml}$ (antiviral activity) & S protein & Kumaki et al. (2011) \\
\hline 127 & SARS-CoV-2 & Kobophenol A & $1.81 \mu \mathrm{M}$ & S protein & Gangadevi et al. (2021) \\
\hline 128 & SARS-CoV & (-)-Catechin gallate & $0.05 \mu \mathrm{g} / \mathrm{ml}$ & $\mathrm{N}$ protein & Roh (2012) \\
\hline 24 & SARS-CoV & (-)-Gallocatechin gallate & $0.05 \mu \mathrm{g} / \mathrm{ml}$ & $\mathrm{N}$ protein & Roh (2012) \\
\hline
\end{tabular}

among which quercetin (22), epigallocatechin gallate (23), and gallocatechin gallate (24) showed inhibitory effects on SARS-CoV $3 \mathrm{CL}^{\text {pro }}$ with $\mathrm{IC}_{50}$ values of $73.00,73.00$, and $47.00 \mu \mathrm{M}$, respectively (Nguyen et al., 2012). Through screening a natural product library consisting of 720 compounds and evaluating extracts of several types of tea, including green tea, oolong tea, Puer tea, and black tea, three natural products-tannic acid (25, $\left.\mathrm{IC}_{50}=3.00 \mu \mathrm{M}\right)$, 3-isotheaflavin-3-gallate $\left(\mathbf{2 6}, \mathrm{IC}_{50}=7.00 \mu \mathrm{M}\right)$, and theaflavin-3, $3^{\prime}$-digallate $\left(27, \mathrm{IC}_{50}=9.50 \mu \mathrm{M}\right)$-were found to be SARS-CoV 3CL ${ }^{\text {pro }}$ inhibitors (Chen et al., 2005). Wen et al. evaluated the anti-SARS-CoV activity of 221 phytocompounds using a cell-based assay measuring SARS-CoV-induced cytopathogenic effect on Vero E6 cells and found that 22 compounds were potent inhibitors at concentrations between 3.30 and $10.00 \mu \mathrm{M}$. Of these, betulinic acid (28), savinin (29), and curcumin (30) displayed potent inhibition toward $3 \mathrm{CL}^{\text {pro }}$ with $\mathrm{IC}_{50}$ values of $10.00,25.00$, and $40.00 \mu \mathrm{M}$, respectively, and the first two blocked the cleavage activity of the $3 \mathrm{CL}^{\text {pro }}$ by 
competitive inhibition (Wen et al., 2007). Curcumin (30, $\mathrm{IC}_{50}=$ $23.50 \mu \mathrm{M}$ ) was used as a positive control in another study which reported that four quinone-methide triterpene derivatives isolated from Tripterygium wilfordii Hook. f., namely, celastrol (31), pristimerin (32), tingenone (33), and iguesterin (34), were identified as inhibitors of SARS-CoV 3CL ${ }^{\text {pro }}$ (Ryu et al., 2010b). Luo et al. reported that several components derived from Rheum palmatum L. showed high inhibitory activity against SARS-CoV $3 \mathrm{CL}^{\text {pro }}$ in in vitro assay. The most active among them, RH121 (35), had an $\mathrm{IC}_{50}$ of $13.76 \mu \mathrm{g} / \mathrm{ml}$, and the inhibition rate was up to 96\% (Luo et al., 2009). Jo et al. applied a flavonoid library to screen and identify herbacetin (36), rhoifolin (37), and pectolinarin (38) as prominent inhibitors blocking the activity of SARS-CoV $3 \mathrm{CL}^{\text {pro }}$ with $\mathrm{IC}_{50}$ values of $33.17,27.45$, and $37.78 \mu \mathrm{M}$, respectively (Jo et al., 2020). In addition, the same author reported that herbacetin (36), isobavachalcone (39), quercetin-3- $\beta$-D-glucoside (40), and helichrysetin (41) were inhibitors against MERS-CoV $3 \mathrm{CL}^{\text {pro }}$ with $\mathrm{IC}_{50}$ values of $40.59,35.85,37.03$, and $67.04 \mu \mathrm{M}$, respectively (Jo et al., 2019).

With the SARS-CoV-2 outbreak, a lot of effort has been devoted to the discovery of natural bioactive molecules against SARS-CoV-2. Quercetin (22), a well-known flavonoid reported as an anti-SARS-CoV natural product, was identified to inhibit $3 \mathrm{CL}^{\text {pro }}$ of SARS-CoV-2 with an inhibition constant $\mathrm{K}_{\mathrm{i}}$ of $7.00 \mu \mathrm{M}$ in an experimental screening of a small chemical library (Abian et al., 2020). Shuanghuanglian preparation is a traditional Chinese medicine with a long history in treating respiratory tract infection in China, and it received widespread attention after the SARS-CoV-2 pandemic. Su et al. recently reported that the oral liquid of Shuanghuanglian, the lyophilized powder of Shuanghuanglian for injection, and their bioactive components exhibited dose-dependent inhibition against the SARS-CoV-2 $3 \mathrm{CL}^{\text {pro }}$ and the replication of SARSCoV-2 in Vero E6 cells. Among these bioactive components, baicalin (42) and baicalein (43) were identified as the first noncovalent and non-peptidomimetic inhibitors of SARS-CoV-2 $3 \mathrm{CL}^{\text {pro }}$, which blocked the cleavage activity of SARS-CoV-2 $3 \mathrm{CL}^{\text {pro }}$ with $\mathrm{IC}_{50}$ values of 6.41 and $0.94 \mu \mathrm{M}$, as well as showing potent antiviral activities in a cell-based system. Furthermore, the crystal complex structure of SARS-CoV-2 $3 \mathrm{CL}^{\text {pro }}$ and baicalein showed that this small flavonoid occupied the core substrate-binding pocket by interacting with two catalytic residues, the crucial S1/S2 subsites and the oxyanion loop, thereby blocking the activity of $3 \mathrm{CL}^{\text {pro }}$ by competitive inhibition (Su et al., 2020). Tannic acid (25) was recently reported to directly interact with SARS-CoV-2 $3 \mathrm{CL}^{\text {pro }}$ with a dissociation constant $\left(\mathrm{K}_{\mathrm{D}}\right)$ of $1.10 \mu \mathrm{M}$ and inhibited $3 \mathrm{CL}^{\text {pro }}$ with an $\mathrm{IC}_{50}$ of $13.40 \mu \mathrm{M}$ (Wang et al., 2020). Additionally, a similar observation of tannic acid (25) with anti-SARS-CoV-2 3CL ${ }^{\text {pro }}$ activity $\left(\mathrm{IC}_{50}=2.10 \mu \mathrm{M}\right)$ was repeatedly reported in another study that also identified hematoporphyrin $\left(52, \mathrm{IC}_{50}=3.90 \mu \mathrm{M}\right)$ as a potent inhibitor against SARS-CoV-2 $3 \mathrm{CL}^{\text {pro }}$ (Coelho et al., 2020). Andrographolide (53), a lactone diterpenoid compound highly abundant in leaves of Andrographis paniculata (Burm. f.) Nees, was reported to suppress $3 \mathrm{CL}^{\text {pro }}$ activities of both SARS$\mathrm{CoV}$ and SARS-CoV-2 with $\mathrm{IC}_{50}$ values of 5.00 and $15.05 \mu \mathrm{M}$. Mass spectrometry (MS) and molecular modeling analysis suggested that andrographolide formed a covalent bond with the active site Cys145 and occupied the catalytic pockets of both viral 3CL ${ }^{\text {pro }}$ s (Shi et al., 2020). In addition, Raj et al. used in silico and in vitro experiments to determine anti-SARS-CoV-2 activities of a series of cannabinoids (CBDs) and identified $\Delta^{9}$ tetrahydrocannabinol (54) and cannabidiol (55) as effective agents against SARS-CoV-2 with $\mathrm{IC}_{50}$ values of 10.25 and $7.91 \mu \mathrm{M}$. Molecular dynamic simulation and density functional theory showed the two compounds formed stable conformations with the active binding pocket of SARS-CoV-2 3CL ${ }^{\text {pro }}$ (Raj et al., 2021). Khan et al. employed similar approaches and reported that kaempferol (56) had an anti-SARS-CoV-2 activity with an $\mathrm{IC}_{50}$ value of $34.46 \mu \mathrm{M}$ in in vitro assay and targeted SARS-CoV-2 $3 \mathrm{CL}^{\text {pro }}$ (Khan et al., 2021).

Another protease, $\mathrm{PL}^{\text {pro }}$, is also regarded as an ideal anti-CoV drug target, and a lot of natural inhibitors targeting this protease have been reported (II, Figure 2). Among these bioactive molecules, some have inhibitory activity against both $\mathrm{PL}^{\text {pro }}$ and $3 \mathrm{CL}^{\text {pro }}$, although most are also somewhat selective (Figure $3 \mathbf{B}$ ). Park et al. published several excellent articles reporting a range of natural bioactive molecules that inhibited both $\mathrm{PL}^{\text {pro }}$ and $3 \mathrm{CL}^{\text {pro }}$ (Park et al., 2012b; Park et al., 2012c; Park et al., 2016; Park et al., 2017). In 2012, they reported nine diarylheptanoids from Alnus japonica (Thunb.) Steud. and evaluated their inhibitory activities against SARS-CoV PL ${ }^{\text {pro }}$ and $3 \mathrm{CL}^{\text {pro }}$ using in vitro assays, and six of these compounds selectively exhibited stronger inhibitory activities against $\mathrm{PL}^{\mathrm{pro}}$ than $3 \mathrm{CL}^{\text {pro }}$. Hirsutenone (57) displayed the most potent $\mathrm{PL}^{\text {pro }}$ inhibitory activity with an $\mathrm{IC}_{50}$ value of $4.10 \mu \mathrm{M}$, similar to positive control curcumin $\left(30, \mathrm{IC}_{50}=5.70 \mu \mathrm{M}\right)$ (Park et al., 2012b). They reported that seven tanshinones derived from Salvia miltiorrhiza Bunge exhibited excellent inhibitory activities against both $\mathrm{PL}^{\text {pro }}$ and $3 \mathrm{CL}^{\text {pro }}$ of SARS-CoV in the same year. Nevertheless, these extract components showed stronger activities against $\mathrm{PL}^{\text {pro }}$ than $3 \mathrm{CL}^{\text {pro }}$, of which cryptotanshinone (66) had the lowest $\mathrm{IC}_{50}$ value of $0.80 \mu \mathrm{M}$ against SARS-CoV PL ${ }^{\text {pro }}$ (Park et al., 2012c). Using cell-free and cell-based assays, the inhibitory activities of 13 constituents from Angelica keiskei (Miq.) Koidz. against SARS-CoV proteases were determined, which showed that chalcones were potent inhibitors against $\mathrm{PL}^{\text {pro }}$ and $3 \mathrm{CL}^{\text {pro }}$ of SARS-CoV. Among them, xanthoangelol E (75) exhibited the most potent inhibitory activities against $\mathrm{PL}^{\text {pro }}$ and $3 \mathrm{CL}^{\text {pro }}$ with $\mathrm{IC}_{50}$ values of 1.20 and $11.40 \mu \mathrm{M}$ (Park et al., 2016). Moreover, 10 polyphenols from Broussonetia papyrifera (L.) L'Hér. ex Vent. and four natural products, namely, isoliquiritigenin (89), kaempferol (56), quercetin (22), and quercetin- $\beta$-galactoside (6), were identified as inhibitors against both $\mathrm{PL}^{\text {pro }}$ and $3 \mathrm{CL}^{\text {pro }}$ of SARS-CoV or MERS-CoV. Similar to their previous studies, all bioactive molecules were more potent against $\mathrm{PL}^{\text {pro }}$ than $3 \mathrm{CL}^{\text {pro }}$. The most potent inhibitor was papyriflavonol A (82), which presented anti-SARS-CoV $\mathrm{PL}^{\text {pro }}$ activity with an $\mathrm{IC}_{50}$ of $3.70 \mu \mathrm{M}$ (Park et al., 2017). In addition to these excellent studies of this team, Chen et al. recently reported ginkgolic acid (90) and anacardic acid (91) as potent covalent inhibitors of both $\mathrm{PL}^{\text {pro }}$ and $3 \mathrm{CL}^{\text {pro }}$ of SARS-CoV-2, and the two compounds showed inhibitory activities against SARS-CoV-2 replication in vitro at non-toxic concentrations (Chen et al., 2021). 

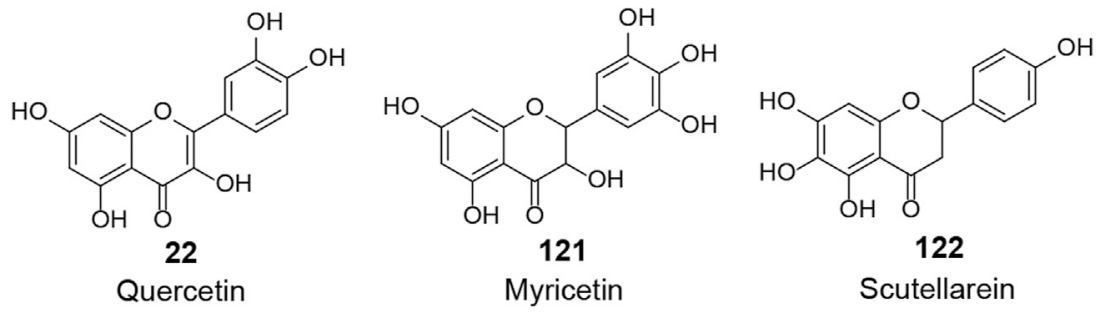

FIGURE 4 | Chemical structure of different natural compounds targeting viral helicase (IV).

Some other studies only reported on natural bioactive molecules that inhibited $\mathrm{PL}^{\text {pro }}$ (Figure 3C). Six cinnamic amides derived from Tribulus terrestris L. fruits exhibited inhibitory activities against SARS-CoV PL ${ }^{\text {pro }}$, of which terrestrimine (97) was the most potent inhibitor with an $\mathrm{IC}_{50}$ of $15.80 \mu \mathrm{M}$ (Song et al., 2014). Cho et al. isolated 12 compounds from Paulownia tomentosa (Thunb.) Steud. fruits, including 5 novel geranylated flavonoid derivatives containing an unusual 3,4-dihydro- $2 \mathrm{H}$-pyran moiety. All derived components dosedependently inhibited $\mathrm{PL}^{\text {pro }}$ with an $\mathrm{IC}_{50}$ range of 5.00-14.40 $\mu \mathrm{M}$, and the 3,4-dihydro-2H-pyran moiety allowed them to inhibit $\mathrm{PL}^{\text {pro }}$ more strongly, especially tomentin E (102) with an $\mathrm{IC}_{50}$ of $5.00 \mu \mathrm{M}$ (Cho et al., 2013). Moreover, six aromatic compounds from Psoralea corylifolia (L.) seeds were identified as potent inhibitors against SARS-CoV $\mathrm{PL}^{\text {pro }}$. Of these bioactive molecules, isobavachalcone $\left(\mathbf{1 1 2}, \mathrm{IC}_{50}=7.30 \mu \mathrm{M}\right)$ and psoralidin (114, $\left.\mathrm{IC}_{50}=4.2 \mu \mathrm{M}\right)$ were the two most promising compounds that inhibit $\mathrm{PL}^{\text {pro }}$ by reversible mixed type I mechanisms, which meant that the compounds preferred to interact with the free enzyme as opposed to the enzyme-substrate complex (Kim et al., 2014).

\section{Replicase-Transcriptase Complex Proteins}

As previously described, the RTC plays a dominant role in generating new genomic and sgRNAs, which are responsible for synthesizing various components of new viruses. RdRp is the core component of RTC and has been considered as an attractive drug target. Despite the development of several well-known drug molecules, such as remdesivir, ribavirin, and favipiravir, as RdRp inhibitors, a few studies have reported natural biomolecular inhibitors against $\mathrm{RdRp}$ (III, Table 1). Fung et al. reported a randomized, doubleblind, placebo-controlled clinical trial result of a Chinese herbal formula named Kwan Du Bu Fei Dang (KDBFD), thought to be a potent anti-SARS-CoV agent. Further, they determined the anti-RdRp activities of KDBFD extract (116) and the extracts of other four traditional Chinese medicines, namely, Houttuynia cordata Thunb. extract (117), Ganoderma lucidum (Leyss. ex Fr.) Karst. extract (118), Coriolus versicolor (L. ex Fr.) Quel. extract (119), and Sinomenium acutum (Thunb.) Rehder \& E. H. Wilson extract (120). The research indicated that these extracts all inhibited SARS-CoV RdRp in a dose-dependent manner with $\mathrm{IC}_{50}$ values ranging between 41.90 and $471.30 \mu \mathrm{g} / \mathrm{ml}$ (Fung et al., 2011).
NTPase/helicase is also essential for viral replication and represents a potential target against coronaviruses. Several flavonoids were determined as inhibitors of NTPase/helicase (IV, Figure 4). Quercetin (22) was reported in several studies as an effective anti-SARS-CoV agent, and, as previously mentioned, it showed potent inhibitory activities against several targets of interest. Lee et al. investigated aryl diketoacids and its bioisostere dihydroxychromone derivatives to reveal the structure activity relationship of such compounds to selectively inhibit the duplex DNA-unwinding activity of SARS$\mathrm{CoV}$ NTPase/helicase. In their study, quercetin $\left(\mathbf{2 2}, \mathrm{IC}_{50}=\right.$ $8.10 \mu \mathrm{M})$ was indicated to selectively inhibit the duplex DNAunwinding activity in the micromolar range (Lee et al., 2009a; Lee et al., 2009b). What is more, this team introduced arylmethyl substituent at the $7-\mathrm{OH}$ position of quercetin by chemical synthesis, resulting in a significant increase in inhibitory activity against SARS-CoV helicase. Of these, 4- $\mathrm{ClPhCH}_{2}$, 3$\mathrm{ClPhCH}_{2}$, and $3-\mathrm{CNPhCH}_{2}$ derivatives exhibited inhibitory activity against helicase with an $\mathrm{IC}_{50}$ range of $2.70-5.20 \mu \mathrm{M}$ (Park et al., 2012a). However, another two flavonoids, myricetin (121) and scutellarein (122), were also reported to inhibit SARS-CoV Nsp13 by affecting its ATPase activity, not the unwinding activity, with $\mathrm{IC}_{50}$ values of 2.71 and $0.86 \mu \mathrm{M}$, respectively.

\section{Structural Proteins}

Structural proteins are essential for viral morphology and life activities. Among the four structural proteins, the $\mathrm{S}$ protein is the most prominent potential target for anti-CoV drugs, because of its crucial role in virus attachment and entry through specific binding to the cellular receptor as well as conformational changes and proteolysis. Several natural products have been reported to exhibit anti-SARS-CoV activities by inhibiting the activity of the $S$ protein or interfering with its interaction with ACE2 (V, Figure 5). Using frontal affinity chromatography-mass spectrometry (FAC/MS) and pseudotyped virus infection assay, Yi et al. screened 121 herbs used in traditional Chinese medicine and identified tetra-O-galloyl- $\beta$-D-glucose (TGG, 123) and luteolin (21), with significant affinity to the S2 protein (Asn733 to Gln 1190 of the SARS-CoV S protein), as agents against SARS$\mathrm{CoV}$ with $\mathrm{EC}_{50}$ values of 4.50 and $10.60 \mu \mathrm{M}$, respectively (Yi et al., 2004). Emodin (124), a bioactive component from Rheum officinale Baill. and Polygonum multiflorum Thunb., was reported to significantly block the binding of the $S$ protein 
<smiles>O=C(O[C@H]1C(O)[C@H](OC(=O)c2cc(O)c(O)c(O)c2)[C@H](OC(=O)c2cc(O)c(O)c(O)c2)[C@H]1OC(=O)c1cc(O)c(O)c(O)c1)c1cc(O)c(O)c(O)c1</smiles>

(TGG)<smiles>O=c1cc(-c2ccc(O)c(O)c2)oc2cc(O)cc(O)c12</smiles>

Luteolin<smiles>O=C1c2c(O)cc(O)cc2C(=[18O])c2cc(O)cc(O)c21</smiles>

Emodin

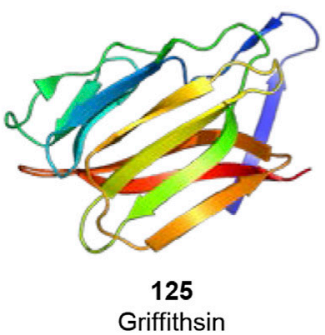

Griffithsin

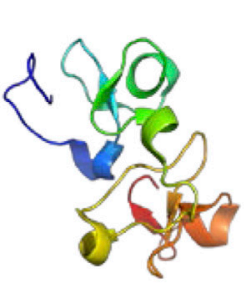

126

Untica dioica agglutinin

(UDA)

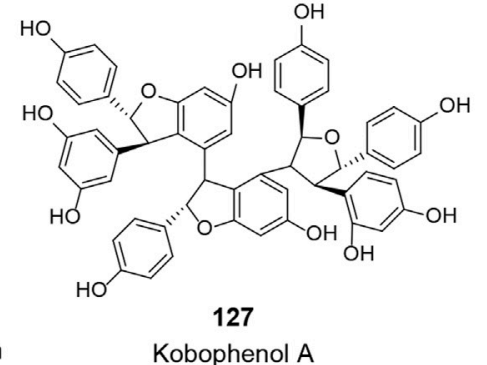

Kobophenol A<smiles>O=C(O[C@H]1Cc2c(O)cc(O)cc2O[C@H]1c1ccc(O)c(O)c1)c1cc(O)c(O)c(O)c1</smiles>

$(-)$-Catechin gallate

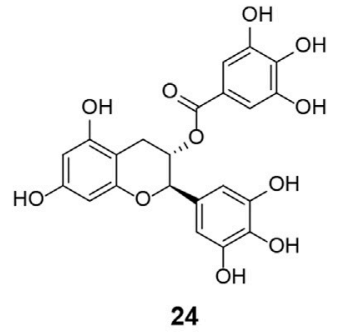

(-)-Gallocatechin gallate

FIGURE 5 | Chemical structure of different natural compounds targeting structural proteins. The S protein (V): tetra-O-galloyl- $\beta$-D-glucose (TGG, 123), luteolin (21), emodin (124), griffithsin (125, PDB: 2GTY), Urtica dioica L. agglutinin (UDA, 126, PDB: 1EN2), and kobophenol A (127). The N protein (VI): (-)-catechin gallate (128) and (-)-gallocatechin gallate (24).

to ACE2 with an $\mathrm{IC}_{50}$ of $200.00 \mu \mathrm{M}$ as well as inhibit the infectivity of the $\mathrm{S}$ protein-pseudotyped retrovirus to Vero E6 cells (Ho et al., 2007). Natural lectins are a class of proteins with specific carbohydrate-binding activity as one or more non-catalytic structural domains can bind specifically and reversibly to monosaccharides or oligosaccharides. Because of the highly glycosylation on the $\mathrm{S}$ protein, lectins are considered as potential anti-CoV candidates (Mitchell et al., 2017). Griffithsin (GRFT, 125, PDB: 2GTY), a lectin isolated from the red algae Griffithsia sp., was identified as a broad-spectrum agent against coronaviruses such as SARS-CoV and MERS-CoV (O'Keefe et al., 2010; Millet et al., 2016). This $12.7 \mathrm{kDa}$ protein was shown to possess three almost identical carbohydrate-binding domains, which allowed GRFT to bind to specific oligosaccharides on envelope glycoproteins and block viral entry (Ziolkowska et al., 2006; Ziolkowska et al., 2007). Isothermal titration calorimetry (ITC) assay showed that GRFT binds to the $S$ protein of SARS-CoV with a stoichiometry of $3: 1$ and a dissociation constant $\left(\mathrm{K}_{\mathrm{D}}\right)$ of $24.90 \mathrm{nM}$. However, it was shown that the binding of GRFT did not interfere with the interaction between the $S$ protein and ACE2 but inhibited in vitro infection of distinct strains of SARS-CoV, including Urbani, Tor-II, CuHK, and Frank strains, with $\mathrm{EC}_{50}$ values ranging between 48.00 and $94.00 \mathrm{nM}$ (O'Keefe et al., 2010). Urtica dioica L. agglutinin (UDA, 126, PDB: 1EN2), an $8.7 \mathrm{kDa}$ plant monomeric lectin, was reported to inhibit the viral replication of distinct strains of SARS-CoV with an $\mathrm{IC}_{50}$ range of $0.60-2.60 \mu \mathrm{g} / \mathrm{ml}$ in Vero 76 cells. In this study, UDA was also found to inhibit SARS-CoV replication in a lethal SARS-CoV BALB/c mouse model and neutralize the virus infectivity by binding to the $S$ protein (Kumaki et al., 2011). In addition, Kobophenol A (127), a bioactive molecule from Caragana sinica (Buc'hoz) Rehder, was recently identified as a potential inhibitor that hinders the interaction between the ACE2 and the S protein in vitro with an $\mathrm{IC}_{50}$ of $1.81 \mu \mathrm{M}$ and inhibits the viral infection of SARS-CoV-2 in cells with an $\mathrm{EC}_{50}$ of $71.60 \mu \mathrm{M}$ (Gangadevi et al., 2021).

The $\mathrm{N}$ protein plays a vital role in virion assembly by enveloping the entire genomic RNA and participating in viral RNA synthesis. The $\mathrm{N}$ protein is also a major pathological determinant in the host and is important for early virus detection and disease diagnosis. Due to its crucial role, the $\mathrm{N}$ protein is also considered an important anti-CoV target (VI, Figure 5). Using a quantum dots-conjugated RNA oligonucleotide system, which simulated the direct binding of the viral RNA to the $\mathrm{N}$ protein on a designed biochip, Roh et al. screened 23 polyphenolic compounds to investigate potential inhibitors of the SARS-CoV $\mathrm{N}$ protein. (-)-Catechin gallate (128) and (-)-gallocatechin gallate (24) were found to inhibit the $\mathrm{N}$ protein binding to the RNA oligonucleotide in a concentration-dependent manner at $0.005 \mu \mathrm{g} /$ $\mathrm{ml}$ or more. At the $0.05 \mu \mathrm{g} / \mathrm{ml}$ concentration, these two compounds displayed more than $40 \%$ inhibitory activity on the designed biochip (Roh, 2012). 
<smiles>O=c1cc(-c2ccc(O)cc2)oc2cc(O)cc(O)c12</smiles>

20

Apigenin<smiles>O=c1cc(-c2ccc(O)c(O)c2)oc2cc(O)cc(O)c12</smiles>

21

Luteolin<smiles>O=c1c(OC2OCC([18OH])C(O)C2O)c(-c2ccc(O)cc2)oc2cc(O)cc(O)c12</smiles>

Kaempferol-3-O- $\alpha$ -arabinopyranoside<smiles>O=c1c(OC2OC(CO)C(O)C(O)C2O)c(-c2ccc(O)cc2)oc2cc(O)cc(O)c12</smiles>

Kaempferol-3-O- $\beta$ -galactopyranoside<smiles>O=c1c(OC2OCC(O)C(O)C2O)c(-c2ccc(O)c(O)c2)oc2cc(O)cc(O)c12</smiles>

131

Quercetin-3-O- $\alpha$ -arabinopyranoside<smiles>O=c1cc(-c2ccc(O)c(O)c2)oc2cc(OC3OC(CO)[C@@H](O)[C@H](O)[C@H]3O)cc(O)c12</smiles>

132

Luteolin-7-O- $\beta$

-glucopyranoside<smiles>N[C@@H](CCN[C@@H](CCN1CCC1C(=O)O)CC(=O)O)C(=O)O</smiles>

133

Nicotianamine

FIGURE 6 | Chemical structure of different natural compounds targeting host ACE2 (VII).

TABLE 2 | Natural compounds targeting host proteins: ACE2 (VII), TMPRSS2 (VIII), and CatL (IX).

\section{No}

20

21

129

130

131

132

133

134

25

32

135

136

137

138

139

140

141

\section{Compound}

\section{Apigenin}

Luteolin

Kaempferol-3-O- $\alpha$-arabinopyranoside

Kaempferol-3-O- $\beta$-galactopyranoside

Quercetin-3-O- $\alpha$-arabinopyranoside

Luteolin-7-O- $\beta$-glucopyranoside

Nicotianamine

Aprotinin

Tannic acid

Celastrol

Leupeptin

Gallinamide A

Panduratin A

Nicolaidesin C

Grassypeptolide A

Grassypeptolide B

Grassypeptolide C
$\mathrm{IC}_{50}$

$280.00 \mu \mathrm{M}$

$290.00 \mu \mathrm{M}$

$320.00 \mu \mathrm{M}$

$260.00 \mu \mathrm{M}$

$310.00 \mu \mathrm{M}$

$280.00 \mu \mathrm{M}$

$84.00 \mathrm{nM}$

No data

$2.31 \mu \mathrm{M}$

No data

No data

$5.00 \mathrm{nM}$

$1.50 \mu \mathrm{M}$

$1.00 \mu \mathrm{M}$

$14.00 \mu \mathrm{M}$

$21.30 \mu \mathrm{M}$

$20.40 \mu \mathrm{M}$
Target

ACE2

ACE2

ACE2

ACE2

ACE2

ACE2

ACE2

TMPRSS2

TMPRSS2

TMPRSS2

Cathepsin L

Cathepsin L

Cathepsin L

Cathepsin L

Cathepsin L

Cathepsin L

Cathepsin L
References

Loizzo et al. (2007)

Loizzo et al. (2007)

Loizzo et al. (2007)

Loizzo et al. (2007)

Loizzo et al. (2007)

Loizzo et al. (2007)

Takahashi et al. (2015)

Shen et al. (2017)

Wang et al. (2020)

(Fernandez-Quintela et al., 2020; Habtemariam et al., 2020) (Salminen and Gottesman, 1990; Nishimura et al., 1995)

Miller et al. (2014)

Deb Majumdar et al. (2011)

Deb Majumdar et al. (2011)

Kwan et al. (2014)

Kwan et al. (2014)

Kwan et al. (2014)

\section{Host Proteins}

During SARS-CoV-2 infection of human cells, some important host proteins play critical roles, including receptor ACE2 and proteases TMPRSS2/4, CatL, furin, etc. In the drug discovery against SARS-CoV-2, targeting viral proteins may be the most direct and effective strategy. However, the fact that viruses can develop drug resistance cannot be ignored. Therefore, targeting these relevant host proteins is another viable strategy. Of course, the safety of this strategy must be carefully considered and evaluated, while it is encouraging that these host proteins have been well studied as therapeutic targets for other diseases and many of the corresponding inhibitors are already in clinical use or under investigation. In the following sections, natural bioactive molecules targeting host proteins will be displayed according to their different targets.

\section{ACE2}

ACE2 is a type I integral membrane protein with a full length of 805 amino acids, including an $\mathrm{N}$-terminal signal peptide sequence of 17 amino acids and a C-terminal membrane- 

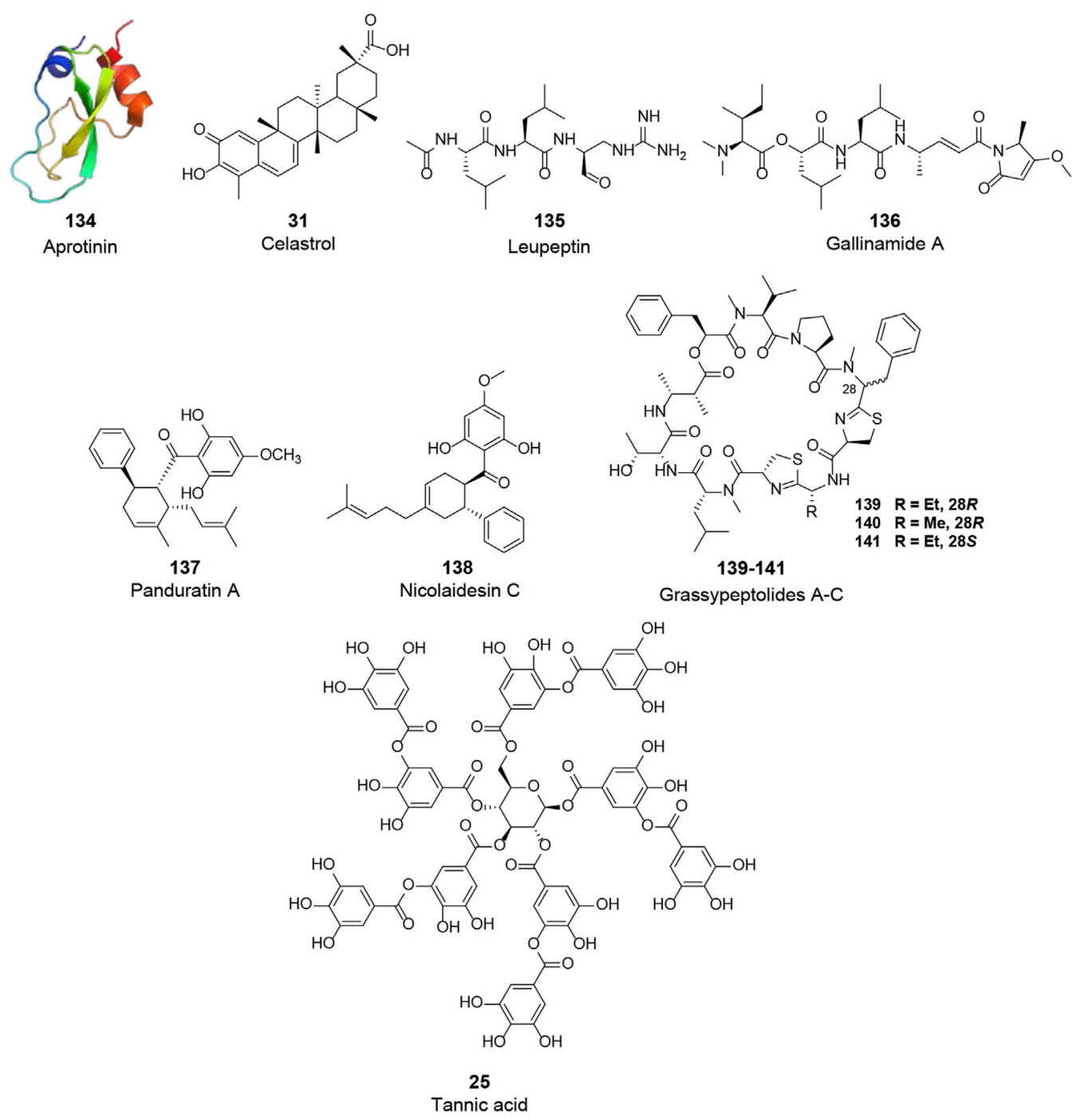

FIGURE 7|Chemical structure of different natural compounds targeting host proteases. TMPRSS2 (VIII): aprotinin (123, PDB: 1BPI), tannic acid (25), and celastrol (31). CatL (IX): leupeptin (135), gallinamide A (136), panduratin A (138), nicolaidesin C (138), and grassypeptolide A-C (139-141).

anchored region as well as a HEXXH-E zinc-binding consensus sequence (Gheblawi et al., 2020). ACE2 has multiple roles, including the negative regulator of the renin-angiotensin system, amino acid transporter, and cellular receptor of SARSCoV and SARS-CoV-2 (Li et al., 2003; Turner et al., 2004; Hashimoto et al., 2012; Yan et al., 2020). As previously described, after SARS-CoV or SARS-CoV-2 invades the body, the $S$ protein binds specifically to ACE2, thus initiating the viral recognition process and entry into the host cell. As a result, drugs that could inhibit or regulate the activity of ACE2 might be potential candidates against SARS-CoV-2. An abundance of natural bioactive molecules have been reported to affect the activity of ACE2 (VII, Figure 6 and Table 2). Several natural products extracted from the leaves of Ailanthus excelsa Roxb., including apigenin (20), luteolin (21), kaempferol-3-O- $\alpha$ arabinopyranoside (129), kaempferol-3-O- $\beta$-galactopyranoside (130), quercetin-3-O- $\alpha$-arabinopyranoside (131), and luteolin-
7-O- $\beta$-glucopyranoside (132), were identified as ACE2 inhibitors with an $\mathrm{IC}_{50}$ range of $260.00-320.00 \mu \mathrm{M}$ in vitro using ACE2 via Elbl and Wagner methods (Loizzo et al., 2007). However, in another study, apigenin (20) was found to up-regulate the expression of ACE2 in the kidney in spontaneously hypertensive rats (Sui et al., 2010). Takahashi et al. synthesized various internally quenched fluorogenic substrates based on the cleavage site of ACE2 and identified Nma-His-Pro-Lys(Dnp) as the most suitable substrate that could be hydrolyzed by recombinant human ACE2. Using the recombinant human ACE2 and Dnp, nicotianamine (133), isolated from Glycine $\max ($ L.) Merr., was identified as a novel ACE2 inhibitor with an $\mathrm{IC}_{50}$ of $84.00 \mathrm{nM}$ (Takahashi et al., 2015).

\section{Host Proteases}

Coronaviruses have evolved multiple strategies for the $\mathrm{S}$ protein hydrolysis, which has been reported to be involved in various host 
proteases, such as TMPRSS2/4, CatL, furin, and trypsin (Millet and Whittaker, 2015). Recently, some of them have been considered potential targets for anti-CoV drugs. In the following, we will present some natural bioactive molecules that have been reported to target TMPRSS2 or CatL for their essential roles in the $S$ protein hydrolysis (Figure 7; Table 2).

TMPRSS2 is type II transmembrane serine protease, which cleaves the $S$ protein after its binding to ACE2, resulting in viral fusion to the cell membrane. Although TMPRSS2 plays an essential role, few natural molecules have been reported to inhibit the activity of TMPRSS2 (VIII, Figure 7). Aprotinin (134, PDB: 1BPI), a polypeptide consisting of 58 amino acid residues purified from bovine lung, was identified as a potential agent against TMPRSS2 (Shen et al., 2017). This polypeptide was shown to inhibit influenza virus replication by inhibiting serine proteases and suppressing the cleavage of influenza virus HA. In addition, it was shown to be effective in mice and human patients and has been approved in Russia as an aerosol for the treatment of patients with mild influenza infections (Ovcharenko and Zhirnov, 1994; Zhirnov et al., 2011). However, more studies are needed to prove its therapeutic activity in coronavirus infections. Tannic acid (25), with inhibitory activities against $3 \mathrm{CL}^{\text {pro }}$ of both SARS-CoV and SARS-CoV-2 as mentioned above, was recently reported to bind to TMPRSS2 with a $\mathrm{K}_{\mathrm{D}}$ of $1.77 \mu \mathrm{M}$ and dose-dependently inhibit TMPRSS2 activity with an $\mathrm{IC}_{50}$ of $2.31 \mu \mathrm{M}$ (Wang et al., 2020). Thus, tannic acid has the promising potential to be a dual inhibitor against SARS-CoV-2. Similarly, celastrol (34), a $3 \mathrm{CL}^{\text {pro }}$ inhibitor, was found to inhibit TMPRSS2 activity. Considering its anti-inflammatory activity by suppressing NF- $\kappa \mathrm{B}$ signaling, celastrol was recently suggested to be a promising drug for the treatment of COVID-19 (Wei and Wang, 2017; Shi et al., 2018; Fernandez-Quintela et al., 2020; Habtemariam et al., 2020).

In addition to TMPRSS2, an endosomal cysteine protease CatL can also hydrolyze and initiate the $\mathrm{S}$ protein activity, allowing the viral membrane fusion via endocytosis. Although CatL is considered dispensable for viral spread and pathogenesis in the infected host compared to TMPRSS2, a variety of natural products have been reported to inhibit this protease and are potential candidates for the treatment of COVID-19 (IX, Figure 7). A pulse-chase experiment in primary cultures of rat hepatocytes showed that the intracellular processing of CatL consisted of two main steps: synthesis of the $39 \mathrm{kDa}$ proenzyme and maturation of the enzyme, in which the $39 \mathrm{kDa}$ proenzyme was processed into 30 and $25 \mathrm{kDa}$ active mature forms of CatL. Leupeptin (135), a non-covalent inhibitor of CatL reported by several early studies, could inhibit the maturation of CatL and lead to intracellular accumulation of the $39 \mathrm{kDa}$ proenzyme (Salminen and Gottesman, 1990; Nishimura et al., 1995). Gallinamide A (136), isolated from cyanobacterium Schizothrix sp., is the most active natural CatL inhibitor reported to date. This bioactive molecule selectively and irreversibly inhibited CatL with an $\mathrm{IC}_{50}$ value of $5.00 \mathrm{nM}$ (Miller et al., 2014). Panduratin A (137) and nicolaidesin C (138), two cyclohexenyl chalcone Diels-Alder natural products, were identified as potential CatL inhibitors with $\mathrm{IC}_{50}$ values of 1.50 and $1.00 \mu \mathrm{M}$, respectively (Deb
Majumdar et al., 2011). Notably, in a recent high-content screening of Thai medicinal plants, panduratin A was identified as an agent against SARS-CoV-2 with an $\mathrm{IC}_{50}$ of $0.81 \mu \mathrm{M}$ and exhibited $99.9 \%$ inhibitory activities against SARS-CoV-2 at $10.00 \mu \mathrm{M}$ (Kanjanasirirat et al., 2020). Although the antiviral mechanism of panduratin A was not fully revealed, its inhibitory activity of CatL might explain this observation. In addition, Kwan et al. investigated the inhibitory activities of several natural products against proteases. Three bisthiazoline containing cyclic depsipeptides from Lyngbya confervoides, grassypeptolides A-C (139-141), were shown to inhibit several proteases, of which these compounds inhibited CatL with $\mathrm{IC}_{50}$ values of $14.00,21.30$, and $20.40 \mu \mathrm{M}$, respectively (Kwan et al., 2014).

\section{Unknown Targets}

In search of anti-CoV agents, many natural bioactive molecules with unknown targets have been reported $(\mathbf{X}$, Figure $\mathbf{8}$ and Table 3). Such natural products, which will be partially but not exclusively listed in this section, possess significant anti-CoV activity with unclear targets and mechanisms. Although more studies are needed to unravel their mechanisms, they remain worthy candidates for the treatment of COVID-19. As the primary effective extract of the well-known phytomedicine liquorice, the antiviral activity of glycyrrhizin (142) has been widely reported ( $\mathrm{Li}$ et al., 2014). The study of Cinatl et al. showed that glycyrrhizin exhibited anti-SARS-CoV activity by inhibiting the viral adsorption, penetration, and replication, and it was more effective when used after the viral adsorption and exhibited the most effective inhibitory activity $\left(\mathrm{EC}_{50}\right.$ of $300 \mathrm{mg} /$ L) when given both during and after the adsorption period (Cinatl et al., 2003). Furthermore, recent studies attempted to explain the antiviral mechanism of glycyrrhizin through pharmacological analysis and in silico methods and suggested a variety of possibilities, including binding to ACE2, downregulating proinflammatory cytokines, inhibiting the accumulation of intracellular reactive oxygen species (ROS), inhibiting thrombin, inhibiting the hyperproduction of airway exudates, and inducing endogenous interferon. Although still insufficient to reveal the exact mechanism, glycyrrhizin may remain a potentially effective agent for the treatment of COVID19 (Bailly and Vergoten, 2020; Luo et al., 2020; Muhseen et al., 2020). Three widely used clinical natural drugs, reserpine (143), aescin (144), and valinomycin (145), derived from Rauvolfia serpentina (L.) Benth. ex Kurz, Aesculus hippocastanum L., and Streptomyces spp., respectively, were reported to inhibit SARS$\mathrm{CoV}$ at micromolar concentration levels (Wu et al., 2004). Considering their excellent bioavailability and safety profile, these clinically approved drugs may be expected to be used directly for COVID-19 treatment. Lycorine (146), an alkaloid from the plants of Amaryllidaceae family, was an outstanding agent against SARS-CoV replication with an $\mathrm{EC}_{50}$ of $15.70 \mathrm{nM}$ in a large in vitro screening and was also proven to inhibit SARSCoV-2 $\left(\mathrm{EC}_{50}=0.31 \mu \mathrm{M}\right)$ in Vero E6 cells (Li et al., 2005; Zhang Y. -N. et al., 2020). Lycorine was reported to effectively inhibit several human coronaviruses, including HCoV-OC43, MERS$\mathrm{CoV}$, and $\mathrm{HCoV}-\mathrm{NL63}$, which suggested that lycorine might be 

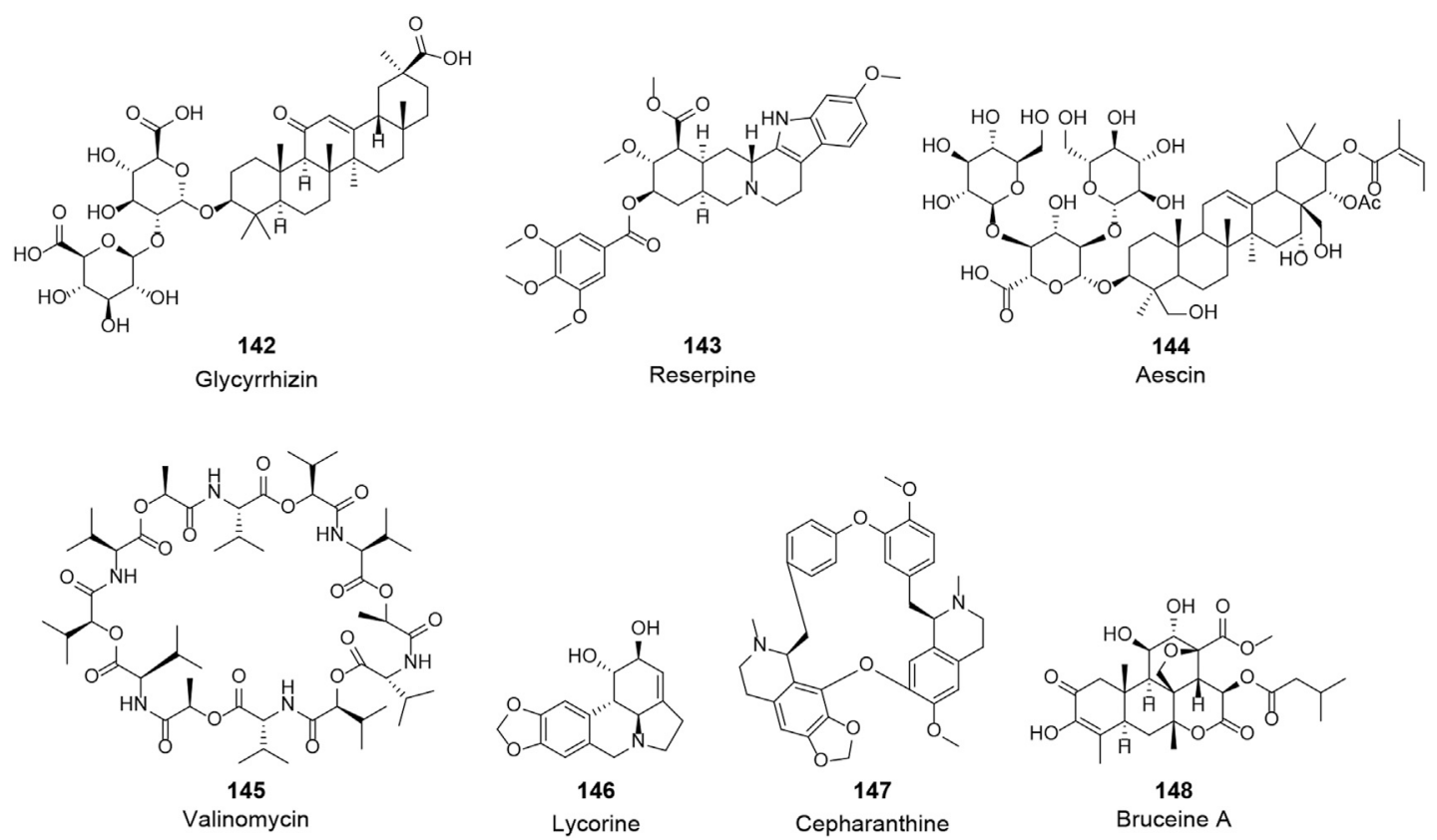

FIGURE 8 | Chemical structure of different natural compounds with significant antiviral activity and without clear targets and mechanisms.

TABLE 3 | Natural compounds with significant anti-CoV activity and without clear targets and mechanisms $(\mathbf{X})$.

\begin{tabular}{lllcc} 
No & Viral strain & Compound & EC $_{\mathbf{5 0}}$ & References \\
\hline 142 & SARS-CoV & Glycyrrhizin & $300.00 \mathrm{mg} / \mathrm{L}$ & Cinatl et al. (2003) \\
143 & SARS-CoV & Reserpine & $3.40 \mu \mathrm{M}$ & Wu et al. (2004) \\
144 & SARS-CoV & Aescin & $6.00 \mu \mathrm{M}$ & Wu et al. (2004) \\
145 & SARS-CoV & Valinomycin & $0.85 \mu \mathrm{M}$ & Wu et al. (2004) \\
146 & SARS-CoV & Lycorine & $15.70 \mathrm{nM}$ & Li et al. (2005) \\
146 & SARS-CoV-2 & Lycorine & $0.31 \mu \mathrm{M}$ & Zhang Y.-N. et al. (2020) \\
147 & SARS-CoV-2 & Cepharanthine & $0.98 \mu \mathrm{M}$ & Fan et al. (2020) \\
148 & SARS-CoV-2 & Bruceine A & $0.011 \mu \mathrm{M}$ & Zhang Z.-R. et al. (2020)
\end{tabular}

a potent agent against coronaviruses (Shen et al., 2019). Recently, several screenings of natural products for antiSARS-CoV-2 have been published. A recent study reported several clinically approved drugs as promising candidates for the treatment of COVID-19, including an alkaloid from the root of Stephania japonica (Thunb.) Miers, cepharanthine (CEP, 147), which is clinically used for leukopenia treatment. The study suggested that CEP could be a wide-spectrum inhibitor of pan-betacoronavirus (Fan et al., 2020). Another cell-based large-scale screening identified 30 natural hits exhibiting suitable anti-SARS-CoV-2 activities with $\mathrm{EC}_{50}$ values ranging between 0.011 and $11.03 \mu \mathrm{M}$. Among these hits, quassinoid derivative bruceine $\mathrm{A}$ (148) was the most potent agent with an $\mathrm{EC}_{50}$ of $0.011 \mu \mathrm{M}$ (Zhang Z.-R. et al., 2020). All of the above natural bioactive molecules have significant anti-CoV activity, and further investigation of their target proteins and mechanisms is of great significance for the development of anti-CoV drugs.

\section{CONCLUSION AND FUTURE PROSPECT}

Natural products have been used as a treasure trove of drug discovery for a long time. These structurally diverse molecules exert a wide range of pharmacological activities, including outstanding antiviral activity. Considerable efforts have been devoted to the development of anti-CoV drugs from natural products, especially in the context of the challenges the world's public health faces, such as the outbreaks of SARS-CoV in 2003 and the current SARS-CoV-2. In order to provide a more systematic understanding of the research on the anti-CoV activity of natural products, we reviewed relevant studies to date, excluding in silico only studies, and summarized numerous natural bioactive molecules based on their protein targets. Most of these natural products are enumerated as inhibitors against SARS-CoV and SARS-CoV-2 and a few molecules that act on MERS-CoV. Among them, flavonoids, alkaloids, terpenoids, and lectins showed encouraging anti$\mathrm{CoV}$ activity, which might provide a large number of promising candidates for the development of anti-CoV drugs and offer potential weapons against SARS-CoV-2 in the present dilemma.

Nonetheless, these studies are often fragmented, and the molecules involved are essentially ubiquitous and represent only a small fraction of the structurally diverse natural products. One corresponding recommendation is to adopt high-throughput screening (HTS) and high-content screening (HCS) to systematically explore natural product resources, especially traditional natural medicines, to discover natural bioactive molecules with excellent anti$\mathrm{CoV}$ activity. In addition, numerous problems still exist, such as the unclear anti-CoV mechanisms, the safety 
issues of natural products, and the drug resistance of coronaviruses. Technologies of structural biology, including nuclear magnetic resonance (NMR), X-ray crystal diffraction, and cryo-electron microscopy (CryoEM), may help to better reveal the anti-CoV mechanisms and targets of effective agents. Researchers can enhance the anti-CoV activity and the safety of natural bioactive molecules through target-based structural modifications and comprehensive safety valuation. Furthermore, to effectively fight against coronaviruses, the combination of natural agents with different targets may be a viable strategy, and the synergy between natural bioactive molecules and conventional drugs should be studied in depth.

In conclusion, there is indeed a long and winding road ahead to develop a feasible anti-CoV drug from natural bioactive lead candidates, which will predictably continue to be invested with more efforts, especially in the current SARS-CoV-2 pandemic.

\section{REFERENCES}

Abian, O., Ortega-Alarcon, D., Jimenez-Alesanco, A., Ceballos-Laita, L., Vega, S., Reyburn, H. T., et al. (2020). Structural Stability of SARS-CoV-2 3CLpro and Identification of Quercetin as an Inhibitor by Experimental Screening. Int. J. Biol. Macromolecules 164, 1693-1703. doi:10.1016/j.ijbiomac.2020.07.235

Adedeji, A. O., Marchand, B., Te Velthuis, A. J. W., Snijder, E. J., Weiss, S., Eoff, R. L., et al. (2012). Mechanism of Nucleic Acid Unwinding by SARS-CoV Helicase. PLoS One 7 (5), e36521. doi:10.1371/journal.pone.0036521

Adnan, M., Alshammari, E., Ashraf, S. A., Patel, K., Lad, K., and Patel, M. (2018a). Physiological and Molecular Characterization of Biosurfactant Producing Endophytic FungiXylaria Regalisfrom the Cones ofThuja Plicataas a Potent Plant Growth Promoter with its Potential Application. Biomed. Res. Int. 2018, 1-11. doi:10.1155/2018/7362148

Adnan, M., Patel, M., Deshpande, S., Alreshidi, M., Siddiqui, A. J., Reddy, M. N., et al. (2020). Effect of Adiantum Philippense Extract on Biofilm Formation, Adhesion with its Antibacterial Activities against Foodborne Pathogens, and Characterization of Bioactive Metabolites: An In Vitro-In Silico Approach. Front. Microbiol. 11, 823. doi:10.3389/fmicb.2020.00823

Adnan, M., Patel, M., Reddy, M. N., and Alshammari, E. (2018b). Formulation, Evaluation and Bioactive Potential of Xylaria Primorskensis Terpenoid Nanoparticles from its Major Compound Xylaranic Acid. Sci. Rep. 8 (1), 1740. doi:10.1038/s41598-018-20237-z

Anand, K., Ziebuhr, J., Wadhwani, P., Mesters, J. R., and Hilgenfeld, R. (2003). Coronavirus Main Proteinase (3CLpro) Structure: Basis for Design of AntiSARS Drugs. Science 300, 1763-1767. doi:10.1126/science.1085658

Arabi, Y. M., Balkhy, H. H., Hayden, F. G., Bouchama, A., Luke, T., Baillie, J. K., et al. (2017). Middle East Respiratory Syndrome. N. Engl. J. Med. 376 (6), 584-594. doi:10.1056/NEJMsr1408795

Arndt, A. L., Larson, B. J., and Hogue, B. G. (2010). A Conserved Domain in the Coronavirus Membrane Protein Tail Is Important for Virus Assembly. J. Virol. 84, 11418-11428. doi:10.1128/jvi.01131-10

Atanasov, A. G., Zotchev, S. B., Zotchev, S. B., Dirsch, V. M., and Supuran, C. T. (2021). Natural Products in Drug Discovery: Advances and Opportunities. Nat. Rev. Drug Discov. 20 (3), 200-216. doi:10.1038/s41573-020-00114-z

Bailly, C., and Vergoten, G. (2020). Glycyrrhizin: An Alternative Drug for the Treatment of COVID-19 Infection and the Associated Respiratory Syndrome?. Pharmacol. Ther. 214, 107618. doi:10.1016/j.pharmthera.2020.107618

Bhardwaj, K., Sun, J., Holzenburg, A., Guarino, L. A., and Kao, C. C. (2006). RNA Recognition and Cleavage by the SARS Coronavirus Endoribonuclease. J. Mol. Biol. 361 (2), 243-256. doi:10.1016/j.jmb.2006.06.021

Bouvet, M., Imbert, I., Subissi, L., Gluais, L., Canard, B., and Decroly, E. (2012). RNA 3'-end Mismatch Excision by the Severe Acute Respiratory Syndrome Coronavirus Nonstructural Protein Nsp10/nsp14 Exoribonuclease Complex. Proc. Natl. Acad. Sci. 109, 9372-9377. doi:10.1073/pnas.1201130109
We hope that researchers can gain insights and valuable information from this review to aid in developing anti-CoV drugs from natural bioactive molecules.

\section{AUTHOR CONTRIBUTIONS}

YL contributed to conception and design of the review. WC wrote the first draft of the manuscript. ZW, YL, and YW wrote sections of the manuscript. All authors contributed to manuscript revision and read and approved the submitted version.

\section{FUNDING}

This work was supported by the National Natural Science Foundation of China (81973240).

Bouvet, M., Lugari, A., Posthuma, C. C., Zevenhoven, J. C., Bernard, S., Betzi, S., et al. (2014). Coronavirus Nsp10, a Critical Co-factor for Activation of Multiple Replicative Enzymes. J. Biol. Chem. 289 (37), 25783-25796. doi:10.1074/ jbc.M114.577353

Ceraolo, C., and Giorgi, F. M. (2020). Genomic Variance of the 2019-nCoV Coronavirus. J. Med. Virol. 92 (5), 522-528. doi:10.1002/jmv.25700

Chan, J. F.-W., Kok, K.-H., Zhu, Z., Chu, H., To, K. K.-W., Yuan, S., et al. (2020). Genomic Characterization of the 2019 Novel Human-Pathogenic Coronavirus Isolated from a Patient with Atypical Pneumonia after Visiting Wuhan. Emerging Microbes \& Infections 9 (1), 221-236. doi:10.1080/ 22221751.2020.1719902

Chang, C.-k., Hou, M.-H., Chang, C.-F., Hsiao, C.-D., and Huang, T.-h. (2014). The SARS Coronavirus Nucleocapsid Protein - Forms and Functions. Antiviral Res. 103, 39-50. doi:10.1016/j.antiviral.2013.12.009

Chellapandi, P., and Saranya, S. (2020). Genomics Insights of SARS-CoV-2 (COVID-19) into Target-Based Drug Discovery. Med. Chem. Res. 29, 1777-1791. doi:10.1007/s00044-020-02610-8

Chen, C.-N., Lin, C. P. C., Huang, K.-K., Chen, W.-C., Hsieh, H.-P., Liang, P.-H., et al. (2005). Inhibition of SARS-CoV 3C-like Protease Activity by Theaflavin3,3'-Digallate (TF3). Evidence-Based Complement. Altern. Med. 2 (2), 209-215. doi:10.1093/ecam/neh081

Chen, L., Li, J., Luo, C., Liu, H., Xu, W., Chen, G., et al. (2006). Binding Interaction of Quercetin-3- $\beta$-Galactoside and its Synthetic Derivatives with SARS-CoV 3CLpro: Structure-Activity Relationship Studies Reveal Salient Pharmacophore Features. Bioorg. Med. Chem. 14 (24), 8295-8306. doi:10.1016/ j.bmc.2006.09.014

Chen, X., Yang, X., Zheng, Y., Yang, Y., Xing, Y., and Chen, Z. (2014). SARS Coronavirus Papain-like Protease Inhibits the Type I Interferon Signaling Pathway through Interaction with the STING-TRAF3-TBK1 Complex. Protein Cell 5 (5), 369-381. doi:10.1007/s13238-014-0026-3

Chen, Y., Cai, H., Pan, J., Xiang, N., Tien, P., Ahola, T., et al. (2009). Functional Screen Reveals SARS Coronavirus Nonstructural Protein Nsp14 as a Novel Cap N7 Methyltransferase. Proc. Natl. Acad. Sci. 106, 3484-3489. doi:10.1073/ pnas.0808790106

Chen, Z., Cui, Q., Cooper, L., Zhang, P., Lee, H., Chen, Z., et al. (2021). Ginkgolic Acid and Anacardic Acid Are Specific Covalent Inhibitors of SARS-CoV-2 Cysteine Proteases. Cell Biosci. 11 (1), 45. doi:10.1186/ s13578-021-00564-x

Cho, J. K., Curtis-Long, M. J., Lee, K. H., Kim, D. W., Ryu, H. W., Yuk, H. J., et al. (2013). Geranylated Flavonoids Displaying SARS-CoV Papain-like Protease Inhibition from the Fruits of Paulownia Tomentosa. Bioorg. Med. Chem. 21 (11), 3051-3057. doi:10.1016/j.bmc.2013.03.027

Chou, K.-C., Wei, D.-Q., and Zhong, W.-Z. (2003). Binding Mechanism of Coronavirus Main Proteinase with Ligands and its Implication to Drug Design against SARS. Biochem. Biophysical Res. Commun. 308 (1), 148-151. doi:10.1016/s0006-291x(03)01342-1 
Christy, M. P., Uekusa, Y., Gerwick, L., and Gerwick, W. H. (2021). Natural Products with Potential to Treat RNA Virus Pathogens Including SARS-CoV-2. J. Nat. Prod. 84 (1), 161-182. doi:10.1021/acs.jnatprod.0c00968

Cinatl, J., Morgenstern, B., Bauer, G., Chandra, P., Rabenau, H., and Doerr, H. (2003). Glycyrrhizin, an Active Component of Liquorice Roots, and Replication of SARS-Associated Coronavirus. The Lancet 361 (9374), 2045-2046. doi:10.1016/s0140-6736(03)13615-x

Cleri, D. J., Ricketti, A. J., and Vernaleo, J. R. (2010). Severe Acute Respiratory Syndrome (SARS). Infect. Dis. Clin. North America 24 (1), 175-202. doi:10.1016/j.idc.2009.10.005

Coelho, C., Gallo, G., Campos, C. B., Hardy, L., and Würtele, M. (2020). Biochemical Screening for SARS-CoV-2 Main Protease Inhibitors. PLoS One 15 (10), e0240079. doi:10.1371/journal.pone.0240079

Deb Majumdar, I., Devanabanda, A., Fox, B., Schwartzman, J., Cong, H., Porco, J. A., Jr., et al. (2011). Synthetic Cyclohexenyl Chalcone Natural Products Possess Cytotoxic Activities against Prostate Cancer Cells and Inhibit Cysteine Cathepsins In Vitro. Biochem. Biophysical Res. Commun. 416 (3-4), 397-402. doi:10.1016/j.bbrc.2011.11.052

Decroly, E., Debarnot, C., Ferron, F., Bouvet, M., Coutard, B., Imbert, I., et al. (2011). Crystal Structure and Functional Analysis of the SARS-Coronavirus RNA Cap 2'-O-Methyltransferase Nsp10/nsp16 Complex. Plos Pathog. 7 (5), e1002059. doi:10.1371/journal.ppat.1002059

Decroly, E., Imbert, I., Coutard, B., Bouvet, M., Selisko, B., Alvarez, K., et al. (2008). Coronavirus Nonstructural Protein 16 Is a Cap-0 Binding Enzyme Possessing (Nucleoside-2' O )-Methyltransferase Activity. J. Virol. 82 (16), 8071-8084. doi:10.1128/JVI.00407-08

Fan, H.-H., Wang, L.-Q., Liu, W.-L., An, X.-P., Liu, Z.-D., He, X.-Q., et al. (2020). Repurposing of Clinically Approved Drugs for Treatment of Coronavirus Disease 2019 in a 2019-novel Coronavirus-Related Coronavirus Model. Chin. Med. J. (Engl) 133 (9), 1051-1056. doi:10.1097/CM9.0000000000000797

Fehr, A. R., and Perlman, S. (2015). "Coronaviruses: An Overview of Their Replication and Pathogenesis," in Coronaviruses: Methods and Protocols. Editors H.J. Maier, E. Bickerton, and P. Britton (New York, NY: Springer New York), 1-23. doi:10.1007/978-1-4939-2438-7_1

Fernández-Quintela, A., Milton-Laskibar, I., Trepiana, J., Gómez-Zorita, S., Kajarabille, N., Léniz, A., et al. (2020). Key Aspects in Nutritional Management of COVID-19 Patients. Jcm 9 (8), 2589. doi:10.3390/jcm9082589

Fung, K. P., Leung, P. C., Tsui, K. W., Wan, C. C., Wong, K. B., Waye, M. Y., et al. (2011). Immunomodulatory Activities of the Herbal Formula Kwan Du Bu Fei Dang in Healthy Subjects: a Randomised, Double-Blind, Placebo-Controlled Study. Hong Kong Med. J. 17 (Suppl. 2), 41-43.

Fung, T. S., and Liu, D. X. (2019). Human Coronavirus: Host-Pathogen Interaction. Annu. Rev. Microbiol. 73, 529-557. doi:10.1146/annurev-micro020518-115759

Gangadevi, S., Badavath, V. N., Thakur, A., Yin, N., De Jonghe, S., Acevedo, O., et al. (2021). Kobophenol A Inhibits Binding of Host ACE2 Receptor with Spike RBD Domain of SARS-CoV-2, a Lead Compound for Blocking COVID-19. J. Phys. Chem. Lett. 12 (7), 1793-1802. doi:10.1021/acs.jpclett.0c03119

Gheblawi, M., Wang, K., Viveiros, A., Nguyen, Q., Zhong, J.-C., Turner, A. J., et al. (2020). Angiotensin-Converting Enzyme 2: SARS-CoV-2 Receptor and Regulator of the Renin-Angiotensin System. Circ. Res. 126 (10), 1456-1474. doi:10.1161/CIRCRESAHA.120.317015

Gordon, D. E., Jang, G. M., Bouhaddou, M., Xu, J., Obernier, K., White, K. M., et al. (2020). A SARS-CoV-2 Protein Interaction Map Reveals Targets for Drug Repurposing. Nature 583 (7816), 459-468. doi:10.1038/s41586-020-2286-9

Habtemariam, S., Nabavi, S. F., Berindan-Neagoe, I., Cismaru, C. A., Izadi, M., Sureda, A., et al. (2020). Should We Try the Antiinflammatory Natural Product, Celastrol, for COVID -19?. Phytotherapy Res. 34 (6), 1189-1190. doi:10.1002/ ptr.6711

Hashimoto, T., Perlot, T., Rehman, A., Trichereau, J., Ishiguro, H., Paolino, M., et al. (2012). ACE2 Links Amino Acid Malnutrition to Microbial Ecology and Intestinal Inflammation. Nature 487 (7408), 477-481. doi:10.1038/ nature11228

Hatakeyama, S., Matsuoka, Y., Ueshiba, H., Komatsu, N., Itoh, K., Shichijo, S., et al. (2008). Dissection and Identification of Regions Required to Form Pseudoparticles by the Interaction between the Nucleocapsid (N) and Membrane (M) Proteins of SARS Coronavirus. Virology 380 (1), 99-108. doi:10.1016/j.virol.2008.07.012
Ho, T., Wu, S., Chen, J., Li, C., and Hsiang, C. (2007). Emodin Blocks the SARS Coronavirus Spike Protein and Angiotensin-Converting Enzyme 2 Interaction. Antiviral Res. 74 (2), 92-101. doi:10.1016/j.antiviral.2006.04.014

Hoffmann, M., Kleine-Weber, H., Schroeder, S., Krüger, N., Herrler, T., Erichsen, S., et al. (2020). SARS-CoV-2 Cell Entry Depends on ACE2 and TMPRSS2 and Is Blocked by a Clinically Proven Protease Inhibitor. Cell 181 (2), 271-280. doi:10.1016/j.cell.2020.02.052

Hu, B., Guo, H., Zhou, P., and Shi, Z.-L. (2020). Characteristics of SARS-CoV-2 and COVID-19. Nat. Rev. Microbiol. 19, 141-154. doi:10.1038/s41579-02000459-7

Huang, C., Lokugamage, K. G., Rozovics, J. M., Narayanan, K., Semler, B. L., and Makino, S. (2011). SARS Coronavirus Nsp1 Protein Induces Templatedependent Endonucleolytic Cleavage of mRNAs: Viral mRNAs Are Resistant to Nsp1-Induced RNA Cleavage. Plos Pathog. 7 (12), e1002433. doi:10.1371/journal.ppat.1002433

Hughes, J., Rees, S., Kalindjian, S., and Philpott, K. (2011). Principles of Early Drug Discovery. Br. J. Pharmacol. 162 (6), 1239-1249. doi:10.1111/j.14765381.2010.01127.x

Hui, D. S. (2017). Epidemic and Emerging Coronaviruses (Severe Acute Respiratory Syndrome and Middle East Respiratory Syndrome). Clin. Chest Med. 38 (1), 71-86. doi:10.1016/j.ccm.2016.11.007

Imbert, I., Guillemot, J.-C., Bourhis, J.-M., Bussetta, C., Coutard, B., Egloff, M.-P., et al. (2006). A Second, Non-canonical RNA-dependent RNA Polymerase in SARS Coronavirus. EMBO J. 25 (20), 4933-4942. doi:10.1038/sj.emboj.7601368

Imbert, I., Snijder, E. J., Dimitrova, M., Guillemot, J.-C., Lécine, P., and Canard, B. (2008). The SARS-Coronavirus PLnc Domain of Nsp3 as a Replication/ transcription Scaffolding Protein. Virus. Res. 133 (2), 136-148. doi:10.1016/ j.virusres.2007.11.017

Ivanov, K. A., Rozanov, M., Bayer, S., Thiel, V., Gorbalenya, A. E., and Ziebuhr, J. (2004a). Major Genetic Marker of Nidoviruses Encodes a Replicative Endoribonuclease. Proc. Natl. Acad. Sci. 101, 12694-12699. doi:10.1073/ pnas. 0403127101

Ivanov, K. A., Thiel, V., Dobbe, J. C., van der Meer, Y., Snijder, E. J., and Ziebuhr, J. (2004b). Multiple Enzymatic Activities Associated with Severe Acute Respiratory Syndrome Coronavirus Helicase. J. Virol. 78 (11), 5619-5632. doi:10.1128/JVI.78.11.5619-5632.2004

Jo, S., Kim, H., Kim, S., Shin, D. H., and Kim, M. S. (2019). Characteristics of Flavonoids as Potent MERS-CoV 3C-like Protease Inhibitors. Chem. Biol. Drug Des. 94 (6), 2023-2030. doi:10.1111/cbdd.13604

Jo, S., Kim, S., Shin, D. H., and Kim, M.-S. (2020). Inhibition of SARS-CoV 3CL Protease by Flavonoids. J. Enzyme Inhib. Med. Chem. 35 (1), 145-151. doi:10.1080/14756366.2019.1690480

Kamitani, W., Narayanan, K., Huang, C., Lokugamage, K., Ikegami, T., Ito, N., et al. (2006). Severe Acute Respiratory Syndrome Coronavirus Nsp1 Protein Suppresses Host Gene Expression by Promoting Host mRNA Degradation. Proc. Natl. Acad. Sci. 103, 12885-12890. doi:10.1073/pnas.0603144103

Kanjanasirirat, P., Suksatu, A., Manopwisedjaroen, S., Munyoo, B., Tuchinda, P., Jearawuttanakul, K., et al. (2020). High-content Screening of Thai Medicinal Plants Reveals Boesenbergia Rotunda Extract and its Component Panduratin A as Anti-SARS-CoV-2 Agents. Sci. Rep. 10 (1), 19963. doi:10.1038/s41598-02077003-3

Khan, A., Heng, W., Wang, Y., Qiu, J., Wei, X., Peng, S., et al. (2021). In Silico and In Vitro Evaluation of Kaempferol as a Potential Inhibitor of the SARS-CoV -2 Main Protease ( 3CLpro ). Phytotherapy Res. 35, 2841-2845. doi:10.1002/ ptr.6998

Kim, D. W., Seo, K. H., Curtis-Long, M. J., Oh, K. Y., Oh, J.-W., Cho, J. K., et al. (2014). Phenolic Phytochemical Displaying SARS-CoV Papain-like Protease Inhibition from the Seeds of Psoralea Corylifolia. J. Enzyme Inhib. Med. Chem. 29 (1), 59-63. doi:10.3109/14756366.2012.753591

Kirchdoerfer, R. N., and Ward, A. B. (2019). Structure of the SARS-CoV Nsp12 Polymerase Bound to Nsp7 and Nsp8 Co-factors. Nat. Commun. 10 (1), 2342. doi:10.1038/s41467-019-10280-3

Kopecky-Bromberg, S. A., Martínez-Sobrido, L., Frieman, M., Baric, R. A., and Palese, P. (2007). Severe Acute Respiratory Syndrome Coronavirus Open reading Frame (ORF) 3b, ORF 6, and Nucleocapsid Proteins Function as Interferon Antagonists. J. Virol. 81 (2), 548-557. doi:10.1128/jvi.01782-06

Kumaki, Y., Wandersee, M. K., Smith, A. J., Zhou, Y., Simmons, G., Nelson, N. M., et al. (2011). Inhibition of Severe Acute Respiratory Syndrome Coronavirus 
Replication in a Lethal SARS-CoV BALB/c Mouse Model by Stinging Nettle Lectin, Urtica Dioica Agglutinin. Antiviral Res. 90 (1), 22-32. doi:10.1016/ j.antiviral.2011.02.003

Kwan, J. C., Liu, Y., Ratnayake, R., Hatano, R., Kuribara, A., Morimoto, C., et al. (2014). Grassypeptolides as Natural Inhibitors of Dipeptidyl Peptidase 8 and T-Cell Activation. Chembiochem 15 (6), 799-804. doi:10.1002/cbic.201300762

Lee, C., Lee, J. M., Lee, N.-R., Jin, B.-S., Jang, K. J., Kim, D.-E., et al. (2009a). Aryl Diketoacids (ADK) Selectively Inhibit Duplex DNA-Unwinding Activity of SARS Coronavirus NTPase/helicase. Bioorg. Med. Chem. Lett. 19 (6), 1636-1638. doi:10.1016/j.bmcl.2009.02.010

Lee, C., Lee, J. M., Lee, N.-R., Kim, D.-E., Jeong, Y.-J., and Chong, Y. (2009b). Investigation of the Pharmacophore Space of Severe Acute Respiratory Syndrome Coronavirus (SARS-CoV) NTPase/helicase by Dihydroxychromone Derivatives. Bioorg. Med. Chem. Lett. 19 (16), 4538-4541. doi:10.1016/j.bmcl.2009.07.009

Lee, N.-R., Kwon, H.-M., Park, K., Oh, S., Jeong, Y.-J., and Kim, D.-E. (2010). Cooperative Translocation Enhances the Unwinding of Duplex DNA by SARS Coronavirus Helicase nsP13. Nucleic Acids Res. 38 (21), 7626-7636. doi:10.1093/nar/gkq647

Lee, Y.-S., Kang, O.-H., Choi, J.-G., Oh, Y.-C., Chae, H.-S., Kim, J. H., et al. (2008). Synergistic Effects of the Combination of Galangin with Gentamicin against Methicillin-Resistant Staphylococcus aureus. J. Microbiol. 46 (3), 283-288. doi:10.1007/s12275-008-0012-7

Lei, J., Kusov, Y., and Hilgenfeld, R. (2018). Nsp3 of Coronaviruses: Structures and Functions of a Large Multi-Domain Protein. Antiviral Res. 149, 58-74. doi:10.1016/j.antiviral.2017.11.001

Li, J.-y., Cao, H.-y., Liu, P., Cheng, G.-h., and Sun, M.-y. (2014). Glycyrrhizic Acid in the Treatment of Liver Diseases: Literature Review. Biomed. Res. Int. 2014, 1-15. doi:10.1155/2014/872139

Li, S., Chen, C., Zhang, H., Guo, H., Wang, H., Wang, L., et al. (2005). Identification of Natural Compounds with Antiviral Activities against SARS-Associated Coronavirus. Antiviral Res. 67 (1), 18-23. doi:10.1016/j.antiviral.2005.02.007

Li, W., Moore, M. J., Vasilieva, N., Sui, J., Wong, S. K., Berne, M. A., et al. (2003). Angiotensin-converting Enzyme 2 Is a Functional Receptor for the SARS Coronavirus. Nature 426 (6965), 450-454. doi:10.1038/nature02145

Lin, C.-W., Tsai, F.-J., Tsai, C.-H., Lai, C.-C., Wan, L., Ho, T.-Y., et al. (2005). AntiSARS Coronavirus 3C-like Protease Effects of Isatis Indigotica Root and PlantDerived Phenolic Compounds. Antiviral Res. 68 (1), 36-42. doi:10.1016/ j.antiviral.2005.07.002

Liu, D. X., Yuan, Q., and Liao, Y. (2007). Coronavirus Envelope Protein: A Small Membrane Protein with Multiple Functions. Cell. Mol. Life Sci. 64 (16), 2043-2048. doi:10.1007/s00018-007-7103-1

Loizzo, M. R., Said, A., Tundis, R., Rashed, K., Statti, G. A., Hufner, A., et al. (2007). Inhibition of Angiotensin Converting Enzyme (ACE) by Flavonoids Isolated fromAilanthus Excelsa (Roxb) (Simaroubaceae). Phytother. Res. 21 (1), 32-36. doi: $10.1002 /$ ptr.2008

Lu, R., Zhao, X., Li, J., Niu, P., Yang, B., Wu, H., et al. (2020). Genomic Characterisation and Epidemiology of 2019 Novel Coronavirus: Implications for Virus Origins and Receptor Binding. The Lancet 395 (10224), 565-574. doi:10.1016/s0140-6736(20)30251-8

Lu, W., Zheng, B.-J., Xu, K., Schwarz, W., Du, L., Wong, C. K. L., et al. (2006). Severe Acute Respiratory Syndrome-Associated Coronavirus 3a Protein Forms an Ion Channel and Modulates Virus Release. Proc. Natl. Acad. Sci. 103 (33), 12540-12545. doi:10.1073/pnas.0605402103

Lugari, A., Betzi, S., Decroly, E., Bonnaud, E., Hermant, A., Guillemot, J.-C., et al. (2010). Molecular Mapping of the RNA Cap 2'-O-Methyltransferase Activation Interface between Severe Acute Respiratory Syndrome Coronavirus Nsp10 and Nsp16*. J. Biol. Chem. 285 (43), 33230-33241. doi:10.1074/jbc.M110.120014

Luo, P., Liu, D., and Li, J. (2020). Pharmacological Perspective: Glycyrrhizin May $\mathrm{Be}$ an Efficacious Therapeutic Agent for COVID-19. Int. J. Antimicrob. Agents 55 (6), 105995. doi:10.1016/j.ijantimicag.2020.105995

Luo, W., Su, X., Gong, S., Qin, Y., Liu, W., Li, J., et al. (2009). Anti-SARS Coronavirus 3C-like Protease Effects of Rheum Palmatum L. Extracts. Biosci. Trends 3 (4), 124-126.

Ma-Lauer, Y., Carbajo-Lozoya, J., Hein, M. Y., Müller, M. A., Deng, W., Lei, J., et al. (2016). p53 Down-Regulates SARS Coronavirus Replication and Is Targeted by the SARS-Unique Domain and PLprovia E3 Ubiquitin Ligase RCHY1. Proc. Natl. Acad. Sci. USA 113 (35), E5192-E5201. doi:10.1073/pnas.1603435113
Mandadi, N. R., Mohd, A., Mousa, M. A., Mohd, S., and Mitesh, P. (2020). Evaluation of Anticancer, Antibacterial and Antioxidant Properties of a Medicinally Treasured Fern Tectaria Coadunata with its Phytoconstituents Analysis by HR-LCMS. Anti-Cancer Agents Med. Chem. 20 (15), 1845-1856. doi:10.2174/1871520620666200318101938

Masters, P. S. (2006). The Molecular Biology of Coronaviruses. Adv. Virus Res. 66, 193-292. doi:10.1016/S0065-3527(06)66005-3

Matsuyama, S., Nao, N., Shirato, K., Kawase, M., Saito, S., Takayama, I., et al. (2020). Enhanced Isolation of SARS-CoV-2 by TMPRSS2-Expressing Cells. Proc. Natl. Acad. Sci. USA 117 (13), 7001-7003. doi:10.1073/pnas.2002589117

McBride, R., Van Zyl, M., and Fielding, B. (2014). The Coronavirus Nucleocapsid Is a Multifunctional Protein. Viruses 6 (8), 2991-3018. doi:10.3390/v6082991

Michel, C. J., Mayer, C., Poch, O., and Thompson, J. D. (2020). Characterization of Accessory Genes in Coronavirus Genomes. Virol. J. 17 (1), 131. doi:10.1186/ s12985-020-01402-1

Miller, B., Friedman, A. J., Choi, H., Hogan, J., McCammon, J. A., Hook, V., et al. (2014). The marine Cyanobacterial Metabolite Gallinamide A Is a Potent and Selective Inhibitor of Human Cathepsin L. J. Nat. Prod. 77 (1), 92-99. doi:10.1021/np400727r

Millet, J. K., Séron, K., Labitt, R. N., Danneels, A., Palmer, K. E., Whittaker, G. R., et al. (2016). Middle East Respiratory Syndrome Coronavirus Infection Is Inhibited by Griffithsin. Antiviral Res. 133, 1-8. doi:10.1016/ j.antiviral.2016.07.011

Millet, J. K., and Whittaker, G. R. (2015). Host Cell Proteases: Critical Determinants of Coronavirus Tropism and Pathogenesis. Virus. Res. 202, 120-134. doi:10.1016/j.virusres.2014.11.021

Mitchell, C. A., Ramessar, K., and O'Keefe, B. R. (2017). Antiviral Lectins: Selective Inhibitors of Viral Entry. Antiviral Res. 142, 37-54. doi:10.1016/ j.antiviral.2017.03.007

Muhseen, Z. T., Hameed, A. R., Al-Hasani, H. M. H., Tahir Ul Qamar, M., and Li, G. (2020). Promising Terpenes as SARS-CoV-2 Spike Receptor-Binding Domain (RBD) Attachment Inhibitors to the Human ACE2 Receptor: Integrated Computational Approach. J. Mol. Liquids 320, 114493. doi:10.1016/j.molliq.2020.114493

Narayanan, K., Huang, C., Lokugamage, K., Kamitani, W., Ikegami, T., Tseng, C.T. K., et al. (2008). Severe Acute Respiratory Syndrome Coronavirus Nsp1 Suppresses Host Gene Expression, Including that of Type I Interferon, in Infected Cells. J. Virol. 82 (9), 4471-4479. doi:10.1128/JVI.02472-07

Nguyen, T. T. H., Woo, H.-J., Kang, H.-K., Nguyen, V. D., Kim, Y.-M., Kim, D.-W., et al. (2012). Flavonoid-mediated Inhibition of SARS Coronavirus 3C-like Protease Expressed in Pichia pastoris. Biotechnol. Lett. 34 (5), 831-838. doi:10.1007/s10529-011-0845-8

Nishimura, Y., Kato, K., Furuno, K., and Himeno, M. (1995). Inhibitory Effect of Leupeptin on the Intracellular Maturation of Lysosomal Cathepsin L in Primary Cultures of Rat Hepatocytes. Biol. Pharm. Bull. 18 (7), 945-950. doi:10.1248/ bpb. 18.945

O'Keefe, B. R., Giomarelli, B., Barnard, D. L., Shenoy, S. R., Chan, P. K. S., McMahon, J. B., et al. (2010). Broad-spectrum In Vitro Activity and In Vivo Efficacy of the Antiviral Protein Griffithsin against Emerging Viruses of the Family Coronaviridae. J. Virol. 84 (5), 2511-2521. doi:10.1128/ JVI.02322-09

Ou, X., Liu, Y., Lei, X., Li, P., Mi, D., Ren, L., et al. (2020). Characterization of Spike Glycoprotein of SARS-CoV-2 on Virus Entry and its Immune Cross-Reactivity with SARS-CoV. Nat. Commun. 11 (1), 1620. doi:10.1038/s41467-020-15562-9

Ovcharenko, A. V., and Zhirnov, O. P. (1994). Aprotinin Aerosol Treatment of Influenza and Paramyxovirus Bronchopneumonia of Mice. Antiviral Res. 23 (2), 107-118. doi:10.1016/0166-3542(94)90038-8

Paraskevis, D., Kostaki, E. G., Magiorkinis, G., Panayiotakopoulos, G., Sourvinos, G., and Tsiodras, S. (2020). Full-genome Evolutionary Analysis of the Novel corona Virus (2019-nCoV) Rejects the Hypothesis of Emergence as a Result of a Recent Recombination Event. Infect. Genet. Evol. 79, 104212. doi:10.1016/ j.meegid.2020.104212

Park, H. R., Yoon, H., Kim, M. K., Lee, S. D., and Chong, Y. (2012a). Synthesis and Antiviral Evaluation of 7-O-Arylmethylquercetin Derivatives against SARSAssociated Coronavirus (SCV) and Hepatitis C Virus (HCV). Arch. Pharm. Res. 35 (1), 77-85. doi:10.1007/s12272-012-0108-9

Park, J.-Y., Jae Jeong, H., Hoon Kim, J., Min Kim, Y., Park, S.-J., Kim, D., et al. (2012b). Diarylheptanoids from Alnus Japonica Inhibit Papain-like Protease of 
Severe Acute Respiratory Syndrome Coronavirus. Biol. Pharm. Bull. 35 (11), 2036-2042. doi:10.1248/bpb.b12-00623

Park, J.-Y., Kim, J. H., Kim, Y. M., Jeong, H. J., Kim, D. W., Park, K. H., et al. (2012c). Tanshinones as Selective and Slow-Binding Inhibitors for SARS-CoV Cysteine Proteases. Bioorg. Med. Chem. 20 (19), 5928-5935. doi:10.1016/ j.bmc.2012.07.038

Park, J.-Y., Ko, J.-A., Kim, D. W., Kim, Y. M., Kwon, H.-J., Jeong, H. J., et al. (2016). Chalcones Isolated fromAngelica Keiskeiinhibit Cysteine Proteases of SARSCoV. J. Enzyme Inhib. Med. Chem. 31 (1), 23-30. doi:10.3109/ 14756366.2014 .1003215

Park, J.-Y., Yuk, H. J., Ryu, H. W., Lim, S. H., Kim, K. S., Park, K. H., et al. (2017). Evaluation of Polyphenols from Broussonetia Papyrifera as Coronavirus Protease Inhibitors. J. Enzyme Inhib. Med. Chem. 32 (1), 504-512. doi:10.1080/14756366.2016.1265519

Patel, M., Ashraf, M. S., Siddiqui, A. J., Ashraf, S. A., Sachidanandan, M., Snoussi, M., et al. (2020). Profiling and Role of Bioactive Molecules from Puntius Sophore (Freshwater/Brackish Fish) Skin Mucus with its Potent Antibacterial, Antiadhesion, and Antibiofilm Activities. Biomolecules 10 (6), 920. doi:10.3390/ biom 10060920

Patel, M., Sachidanandan, M., and Adnan, M. (2019). Serine Arginine Protein Kinase 1 (SRPK1): a Moonlighting Protein with Theranostic Ability in Cancer Prevention. Mol. Biol. Rep. 46 (1), 1487-1497. doi:10.1007/s11033-018-4545-5

Paules, C. I., Marston, H. D., and Fauci, A. S. (2020). Coronavirus Infections-More Than Just the Common Cold. JAMA 323 (8), 707-708. doi:10.1001/ jama.2020.0757

Pfefferle, S., Schöpf, J., Kögl, M., Friedel, C. C., Müller, M. A., Carbajo-Lozoya, J., et al. (2011). The SARS-Coronavirus-Host Interactome: Identification of Cyclophilins as Target for Pan-Coronavirus Inhibitors. Plos Pathog. 7 (10), e1002331. doi:10.1371/journal.ppat.1002331

Pillay, T. S. (2020). Gene of the Month: the 2019-nCoV/SARS-CoV-2 Novel Coronavirus Spike Protein. J. Clin. Pathol. 73 (7), 366-369. doi:10.1136/ jclinpath-2020-206658

Prentice, E., McAuliffe, J., Lu, X., Subbarao, K., and Denison, M. R. (2004). Identification and Characterization of Severe Acute Respiratory Syndrome Coronavirus Replicase Proteins. J. Virol. 78 (18), 9977-9986. doi:10.1128/ JVI.78.18.9977-9986.2004

Raj, V., Park, J. G., Cho, K.-H., Choi, P., Kim, T., Ham, J., et al. (2021). Assessment of Antiviral Potencies of Cannabinoids against SARS-CoV-2 Using Computational and In Vitro Approaches. Int. J. Biol. Macromolecules 168, 474-485. doi:10.1016/j.ijbiomac.2020.12.020

Roh, C. (2012). A Facile Inhibitor Screening of SARS Coronavirus N Protein Using Nanoparticle-Based RNA Oligonucleotide. Ijn 7, 2173-2179. doi:10.2147/ IJN.S31379

Ruch, T. R., and Machamer, C. E. (2012). The Coronavirus E Protein: Assembly and beyond. Viruses 4 (3), 363-382. doi:10.3390/v4030363

Russo, G. L., Tedesco, I., Spagnuolo, C., and Russo, M. (2017). Antioxidant Polyphenols in Cancer Treatment: Friend, Foe or Foil? Semin. Cancer Biol. 46, 1-13. doi:10.1016/j.semcancer.2017.05.005

Russo, M., Moccia, S., Spagnuolo, C., Tedesco, I., and Russo, G. L. (2020). Roles of Flavonoids against Coronavirus Infection. Chemico-Biological Interactions 328, 109211. doi:10.1016/j.cbi.2020.109211

Ryu, Y. B., Jeong, H. J., Kim, J. H., Kim, Y. M., Park, J.-Y., Kim, D., et al. (2010a). Biflavonoids from Torreya Nucifera Displaying SARS-CoV 3CLpro Inhibition. Bioorg. Med. Chem. 18 (22), 7940-7947. doi:10.1016/j.bmc.2010.09.035

Ryu, Y. B., Park, S.-J., Kim, Y. M., Lee, J.-Y., Seo, W. D., Chang, J. S., et al. (2010b). SARS-CoV 3CLpro Inhibitory Effects of Quinone-Methide Triterpenes from Tripterygium Regelii. Bioorg. Med. Chem. Lett. 20 (6), 1873-1876. doi:10.1016/ j.bmcl.2010.01.152

Saberi, A., Gulyaeva, A. A., Brubacher, J. L., Newmark, P. A., and Gorbalenya, A. E. (2018). A Planarian Nidovirus Expands the Limits of RNA Genome Size. Plos Pathog. 14 (11), e1007314. doi:10.1371/journal.ppat.1007314

Salminen, A., and Gottesman, M. M. (1990). Inhibitor Studies Indicate that Active Cathepsin L Is Probably Essential to its Own Processing in Cultured Fibroblasts. Biochem. J. 272 (1), 39-44. doi:10.1042/bj2720039

Schoeman, D., and Fielding, B. C. (2019). Coronavirus Envelope Protein: Current Knowledge. Virol. J. 16 (1), 69. doi:10.1186/s12985-019-1182-0

Seybert, A., Hegyi, A., Siddell, S. G., and Ziebuhr, J. (2000). The Human Coronavirus 229E Superfamily 1 Helicase Has RNA and DNA Duplex-Unwinding
Activities with $5^{\prime}$-to-3' Polarity. RNA 6 (7), 1056-1068, doi:10.1017/ s1355838200000728

Shen, L., Niu, J., Wang, C., Huang, B., Wang, W., Zhu, N., et al. (2019). HighThroughput Screening and Identification of Potent Broad-Spectrum Inhibitors of Coronaviruses. J. Virol. 93 (12), e00023-00019. doi:10.1128/JVI.00023-19

Shen, L. W., Mao, H. J., Wu, Y. L., Tanaka, Y., and Zhang, W. (2017). TMPRSS2: A Potential Target for Treatment of Influenza Virus and Coronavirus Infections. Biochimie 142, 1-10. doi:10.1016/j.biochi.2017.07.016

Shi, K., Chen, X., Xie, B., Yang, S. S., Liu, D., Dai, G., et al. (2018). Celastrol Alleviates Chronic Obstructive Pulmonary Disease by Inhibiting Cellular Inflammation Induced by Cigarette Smoke via the Ednrb/Kng1 Signaling Pathway. Front. Pharmacol. 9, 1276. doi:10.3389/fphar.2018.01276

Shi, T.-H., Huang, Y.-L., Chen, C.-C., Pi, W.-C., Hsu, Y.-L., Lo, L.-C., et al. (2020). Andrographolide and its Fluorescent Derivative Inhibit the Main Proteases of 2019-nCoV and SARS-CoV through Covalent Linkage. Biochem. Biophysical Res. Commun. 533 (3), 467-473. doi:10.1016/j.bbrc.2020.08.086

Siddiqui, A. J., Danciu, C., Ashraf, S. A., Moin, A., Singh, R., Alreshidi, M., et al. (2020). Plants-Derived Biomolecules as Potent Antiviral Phytomedicines: New Insights on Ethnobotanical Evidences against Coronaviruses. Plants 9 (9), 1244. doi:10.3390/plants9091244

Siu, K.-L., Chan, C.-P., Kok, K.-H., Chiu-Yat Woo, P., and Jin, D.-Y. (2014). Suppression of Innate Antiviral Response by Severe Acute Respiratory Syndrome Coronavirus M Protein Is Mediated through the First Transmembrane Domain. Cell Mol Immunol 11 (2), 141-149. doi:10.1038/ cmi.2013.61

Skariyachan, S., Challapilli, S. B., Packirisamy, S., Kumargowda, S. T., and Sridhar, V. S. (2019). Recent Aspects on the Pathogenesis Mechanism, Animal Models and Novel Therapeutic Interventions for Middle East Respiratory Syndrome Coronavirus Infections. Front. Microbiol. 10, 569. doi:10.3389/ fmicb.2019.00569

Song, Y. H., Kim, D. W., Curtis-Long, M. J., Yuk, H. J., Wang, Y., Zhuang, N., et al. (2014). Papain-like Protease (PLpro) Inhibitory Effects of Cinnamic Amides from Tribulus Terrestris Fruits. Biol. Pharm. Bull. 37 (6), 1021-1028. doi:10.1248/bpb.b14-00026

Stadler, K., Masignani, V., Eickmann, M., Becker, S., Abrignani, S., Klenk, H.-D., et al. (2003). SARS - Beginning to Understand a New Virus. Nat. Rev. Microbiol. 1 (3), 209-218. doi:10.1038/nrmicro775

Su, H.-x., Yao, S., Zhao, W.-f., Li, M.-j., Liu, J., Shang, W.-j., et al. (2020). AntiSARS-CoV-2 Activities In Vitro of Shuanghuanglian Preparations and Bioactive Ingredients. Acta Pharmacol. Sin 41 (9), 1167-1177. doi:10.1038/ s41401-020-0483-6

Sui, H., Yu, Q., Zhi, Y., Geng, G., Liu, H., and Xu, H. (2010). [Effects of Apigenin on the Expression of Angiotensin-Converting Enzyme 2 in Kidney in Spontaneously Hypertensive Rats]. Wei Sheng Yan Jiu 39 (6), 693-696.

Sung, W. S., and Lee, D. G. (2008). The Combination Effect of Korean Red Ginseng Saponins with Kanamycin and Cefotaxime against Methicillin-Resistant Staphylococcus aureus. Biol. Pharm. Bull. 31 (8), 1614-1617. doi:10.1248/ bpb.31.1614

Takahashi, S., Yoshiya, T., Yoshizawa-Kumagaye, K., and Sugiyama, T. (2015). Nicotianamine Is a Novel Angiotensin-Converting Enzyme 2 Inhibitor in Soybean. Biomed. Res. 36 (3), 219-224. doi:10.2220/biomedres.36.219

Tanner, J. A., Watt, R. M., Chai, Y.-B., Lu, L.-Y., Lin, M. C., Peiris, J. S. M., et al. (2003). The Severe Acute Respiratory Syndrome (SARS) Coronavirus NTPase/ Helicase Belongs to a Distinct Class of $5^{\prime}$ to $3^{\prime}$ Viral Helicases. J. Biol. Chem. 278 (41), 39578-39582. doi:10.1074/jbc.C300328200

te Velthuis, A. J. W., Arnold, J. J., Cameron, C. E., van den Worm, S. H. E., and Snijder, E. J. (2010). The RNA Polymerase Activity of SARS-Coronavirus Nsp12 Is Primer Dependent. Nucleic Acids Res. 38 (1), 203-214. doi:10.1093/nar/gkp904

te Velthuis, A. J. W., van den Worm, S. H. E., and Snijder, E. J. (2012). The SARSCoronavirus Nsp7+nsp8 Complex Is a Unique Multimeric RNA Polymerase Capable of Both De Novo Initiation and Primer Extension. Nucleic Acids Res. 40 (4), 1737-1747. doi:10.1093/nar/gkr893

Tohya, Y., Narayanan, K., Kamitani, W., Huang, C., Lokugamage, K., and Makino, S. (2009). Suppression of Host Gene Expression by Nsp1 Proteins of Group 2 Bat Coronaviruses. J. Virol. 83 (10), 5282-5288. doi:10.1128/JVI.02485-08

$\mathrm{Tu}, \mathrm{Y}$. (2011). The Discovery of Artemisinin (Qinghaosu) and Gifts from Chinese Medicine. Nat. Med. 17 (10), 1217-1220. doi:10.1038/nm.2471 
Turner, A. J., Hiscox, J. A., and Hooper, N. M. (2004). ACE2: from Vasopeptidase to SARS Virus Receptor. Trends Pharmacol. Sci. 25 (6), 291-294. doi:10.1016/ j.tips.2004.04.001

von Brunn, A., Teepe, C., Simpson, J. C., Pepperkok, R., Friedel, C. C., Zimmer, R., et al. (2007). Analysis of Intraviral Protein-Protein Interactions of the SARS Coronavirus ORFeome. PLoS One 2 (5), e459. doi:10.1371/ journal.pone.0000459

Walls, A. C., Park, Y.-J., Tortorici, M. A., Wall, A., McGuire, A. T., and Veesler, D. (2020). Structure, Function, and Antigenicity of the SARS-CoV-2 Spike Glycoprotein. Cell 181 (2), 281-292. doi:10.1016/j.cell.2020.02.058

Wang, S. C., Chen, Y., Wang, Y. C., Wang, W. J., Yang, C. S., Tsai, C. L., et al. (2020). Tannic Acid Suppresses SARS-CoV-2 as a Dual Inhibitor of the Viral Main Protease and the Cellular TMPRSS2 Protease. Am. J. Cancer Res. 10 (12), $4538-4546$.

Wei, Y., and Wang, Y. (2017). Celastrol Attenuates Impairments Associated with Lipopolysaccharide-Induced Acute Respiratory Distress Syndrome (ARDS) in Rats. J. Immunotoxicology 14 (1), 228-234. doi:10.1080/ 1547691X.2017.1394933

Wen, C.-C., Kuo, Y.-H., Jan, J.-T., Liang, P.-H., Wang, S.-Y., Liu, H.-G., et al. (2007). Specific Plant Terpenoids and Lignoids Possess Potent Antiviral Activities against Severe Acute Respiratory Syndrome Coronavirus. J. Med. Chem. 50 (17), 4087-4095. doi:10.1021/jm070295s

Wrapp, D., Wang, N., Corbett, K. S., Goldsmith, J. A., Hsieh, C.-L., Abiona, O., et al. (2020). Cryo-EM Structure of the 2019-nCoV Spike in the Prefusion Conformation. Science 367 (6483), 1260-1263. doi:10.1126/ science.abb2507

Wrobel, A. G., Benton, D. J., Xu, P., Roustan, C., Martin, S. R., Rosenthal, P. B., et al. (2020). SARS-CoV-2 and Bat RaTG13 Spike Glycoprotein Structures Inform on Virus Evolution and Furin-Cleavage Effects. Nat. Struct. Mol. Biol. 27 (8), 763-767. doi:10.1038/s41594-020-0468-7

Wu, C.-Y., Jan, J.-T., Ma, S.-H., Kuo, C.-J., Juan, H.-F., Cheng, Y.-S. E., et al. (2004). Small Molecules Targeting Severe Acute Respiratory Syndrome Human Coronavirus. Proc. Natl. Acad. Sci. 101 (27), 10012-10017. doi:10.1073/ pnas.0403596101

Wu, F., Zhao, S., Yu, B., Chen, Y.-M., Wang, W., Song, Z.-G., et al. (2020). A New Coronavirus Associated with Human Respiratory Disease in China. Nature 579 (7798), 265-269. doi:10.1038/s41586-020-2008-3

Xia, S., Yan, L., Xu, W., Agrawal, A. S., Algaissi, A., Tseng, C.-T. K., et al. (2019). A Pan-Coronavirus Fusion Inhibitor Targeting the HR1 Domain of Human Coronavirus Spike. Sci. Adv. 5 (4), eaav4580. doi:10.1126/sciadv.aav4580

Xiao, Y., Ma, Q., Restle, T., Shang, W., Svergun, D. I., Ponnusamy, R., et al. (2012). Nonstructural Proteins 7 and 8 of Feline Coronavirus Form a 2:1 Heterotrimer that Exhibits Primer-independent RNA Polymerase Activity. J. Virol. 86 (8), 4444-4454. doi:10.1128/JVI.06635-11

Xu, C., Ke, Z., Liu, C., Wang, Z., Liu, D., Zhang, L., et al. (2020). Systemic In Silico Screening in Drug Discovery for Coronavirus Disease (COVID-19) with an Online Interactive Web Server. J. Chem. Inf. Model. 60 (12), 5735-5745. doi:10.1021/acs.jcim.0c00821

Yan, R., Zhang, Y., Li, Y., Xia, L., Guo, Y., and Zhou, Q. (2020). Structural Basis for the Recognition of SARS-CoV-2 by Full-Length Human ACE2. Science 367, 1444-1448. doi:10.1126/science.abb2762

Yi, L., Li, Z., Yuan, K., Qu, X., Chen, J., Wang, G., et al. (2004). Small Molecules Blocking the Entry of Severe Acute Respiratory Syndrome Coronavirus into Host Cells. J. Virol. 78 (20), 11334-11339. doi:10.1128/JVI.78.20.1133411339.2004

Yin, J., Niu, C., Cherney, M. M., Zhang, J., Huitema, C., Eltis, L. D., et al. (2007). A Mechanistic View of Enzyme Inhibition and Peptide Hydrolysis in the Active
Site of the SARS-CoV 3C-like Peptidase. J. Mol. Biol. 371 (4), 1060-1074. doi:10.1016/j.jmb.2007.06.001

Yu, M.-S., Lee, J., Lee, J. M., Kim, Y., Chin, Y.-W., Jee, J.-G., et al. (2012). Identification of Myricetin and Scutellarein as Novel Chemical Inhibitors of the SARS Coronavirus Helicase, nsP13. Bioorg. Med. Chem. Lett. 22 (12), 4049-4054. doi:10.1016/j.bmcl.2012.04.081

Zang, R., Castro, M. F. G., McCune, B. T., Zeng, Q., Rothlauf, P. W., Sonnek, N. M., et al. (2020). TMPRSS2 and TMPRSS4 Promote SARS-CoV-2 Infection of Human Small Intestinal Enterocytes. Sci. Immunol. 5. doi:10.1126/ sciimmunol.abc3582

Zhang, Y.-N., Zhang, Q.-Y., Li, X.-D., Xiong, J., Xiao, S.-Q., Wang, Z., et al. (2020). Gemcitabine, Lycorine and Oxysophoridine Inhibit Novel Coronavirus (SARSCoV-2) in Cell Culture. Emerging Microbes \& Infections 9 (1), 1170-1173. doi:10.1080/22221751.2020.1772676

Zhang, Z.-R., Zhang, Y.-N., Li, X.-D., Zhang, H.-Q., Xiao, S.-Q., Deng, F., et al. (2020). A Cell-Based Large-Scale Screening of Natural Compounds for Inhibitors of SARS-CoV-2. Sig Transduct Target. Ther. 5 (1), 218. doi:10.1038/s41392-020-00343-Z

Zhirnov, O. P., Klenk, H. D., and Wright, P. F. (2011). Aprotinin and Similar Protease Inhibitors as Drugs against Influenza. Antiviral Res. 92 (1), 27-36. doi:10.1016/j.antiviral.2011.07.014

Zhou, P., Li, H., Wang, H., Wang, L.-F., and Shi, Z. (2012). Bat Severe Acute Respiratory Syndrome-like Coronavirus ORF3b Homologues Display Different Interferon Antagonist Activities. J. Gen. Virol. 93 (Pt 2), 275-281. doi:10.1099/ vir. $0.033589-0$

Ziebuhr, J. (2004). Molecular Biology of Severe Acute Respiratory Syndrome Coronavirus. Curr. Opin. Microbiol. 7 (4), 412-419. doi:10.1016/ j.mib.2004.06.007

Ziebuhr, J. (2005). "The Coronavirus Replicase," in Coronavirus Replication and Reverse Genetics. Editor L. Enjuanes (Berlin, Heidelberg: Springer Berlin Heidelberg), 57-94. doi:10.1007/3-540-26765-4_3

Ziółkowska, N. E., O’Keefe, B. R., Mori, T., Zhu, C., Giomarelli, B., Vojdani, F., et al. (2006). Domain-swapped Structure of the Potent Antiviral Protein Griffithsin and its Mode of Carbohydrate Binding. Structure 14 (7), 1127-1135. doi:10.1016/j.str.2006.05.017

Ziółkowska, N. E., Shenoy, S. R., O’Keefe, B. R., McMahon, J. B., Palmer, K. E., Dwek, R. A., et al. (2007). Crystallographic, Thermodynamic, and Molecular Modeling Studies of the Mode of Binding of Oligosaccharides to the Potent Antiviral Protein Griffithsin. Proteins 67 (3), 661-670. doi:10.1002/prot.21336

Conflict of Interest: The authors declare that the research was conducted in the absence of any commercial or financial relationships that could be construed as a potential conflict of interest.

Publisher's Note: All claims expressed in this article are solely those of the authors and do not necessarily represent those of their affiliated organizations, or those of the publisher, the editors and the reviewers. Any product that may be evaluated in this article, or claim that may be made by its manufacturer, is not guaranteed or endorsed by the publisher.

Copyright (ㄷ 2021 Chen, Wang, Wang and Li. This is an open-access article distributed under the terms of the Creative Commons Attribution License (CC $B Y)$. The use, distribution or reproduction in other forums is permitted, provided the original author(s) and the copyright owner(s) are credited and that the original publication in this journal is cited, in accordance with accepted academic practice. No use, distribution or reproduction is permitted which does not comply with these terms. 